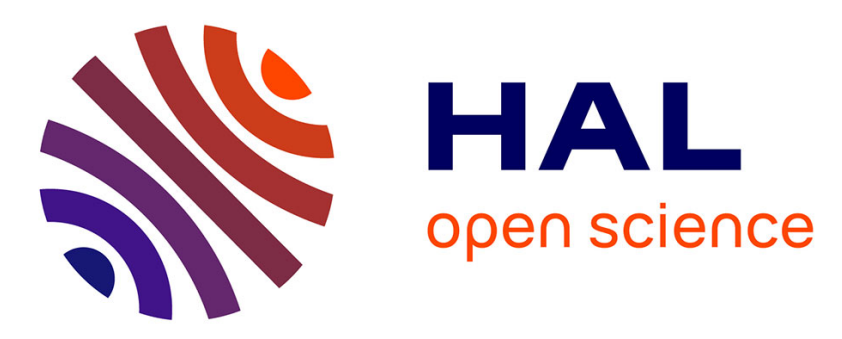

\title{
A priori filtering and LES modeling of turbulent two-phase flows application to phase separation
}

\author{
S. Vincent, M. Tavares, S. Fleau, S. Mimouni, M. Ould-Rouiss, Jean-Luc
}

Estivalezes

\section{- To cite this version:}

S. Vincent, M. Tavares, S. Fleau, S. Mimouni, M. Ould-Rouiss, et al.. A priori filtering and LES modeling of turbulent two-phase flows application to phase separation. Computers and Fluids, In press, 10.1016/j.compfluid.2016.10.021 . hal-01853399

\section{HAL Id: hal-01853399 \\ https://hal.science/hal-01853399}

Submitted on 7 Aug 2018

HAL is a multi-disciplinary open access archive for the deposit and dissemination of scientific research documents, whether they are published or not. The documents may come from teaching and research institutions in France or abroad, or from public or private research centers.
L'archive ouverte pluridisciplinaire HAL, est destinée au dépôt et à la diffusion de documents scientifiques de niveau recherche, publiés ou non, émanant des établissements d'enseignement et de recherche français ou étrangers, des laboratoires publics ou privés. 


\title{
A priori filtering and LES modeling of turbulent two-phase flows Application to phase separation
}

\author{
S. Vincent ${ }^{\dagger, a}$, M. Tavares ${ }^{\dagger}$, Solène Fleau ${ }^{o, \dagger}$, \\ S. Mimouni ${ }^{\circ}$, M. Ould-Rouiss ${ }^{\dagger}$, J.-L. Estivalezes \\ ${ }^{a}$ stephane.vincent@u-pem.fr (corresponding author) \\ † Université Paris-Est Marne-La-Vallée, Laboratoire Modélisation et \\ Simulation Multi Echelle (MSME), UMR CNRS 8208, F-77454, \\ Marne-La-Vallée, France \\ ${ }^{o}$ Electricité de France, R\&D division, F-78400 Chatou, France \\ - ONERA, The French Aerospace Lab, F-31055 Toulouse, France
}

\begin{abstract}
The Large Eddy Simulation (LES) of two-phase flows with resolved scale interfaces is investigated through the a priori filtering of DNS simulations of one-fluid and multifield models. A phase inversion benchmark [1-4] is considered highlighting many coalescence and interface rupture events in a kind of atomization process. The order of magnitude of specific two-phase subgrid LES terms is first considered with the two modeling approaches. Then, different existing models such as Smagorinsky [5], Wale [6], Bardina [7], Mixed [8] and ADM [9] are used to account for two-phase subgrid effects. These models are compared to filtered DNS results.
\end{abstract}

keywords: phase inversion, two-phase flows, a priori filtering, LES modeling, one-fluid model, multi-field approach 


\section{Introduction}

Turbulent two-phase flows occur in many academic and applied fluid mechanics problems such as boiling crisis in nuclear plants, chemical reactors, material coating by plasma projection, bubbles in pipes, wave breaking, oil extraction in porous media, powder and fluidized beds processes, fuel injection in engines to cite a few. Exhaustive presentation of various turbulent multi-phase flows is given for example in [10]. As soon as turbulence and interface interact in a non linear way with macroscopic interfacial deformations inducing ligaments, coalescence or rupture, the experimental characterization of these flows is difficult due to the heterogenous character of the multi-phase medium. Modeling and numerical simulation thus represent an interesting way to study the physical processes that control these flows.

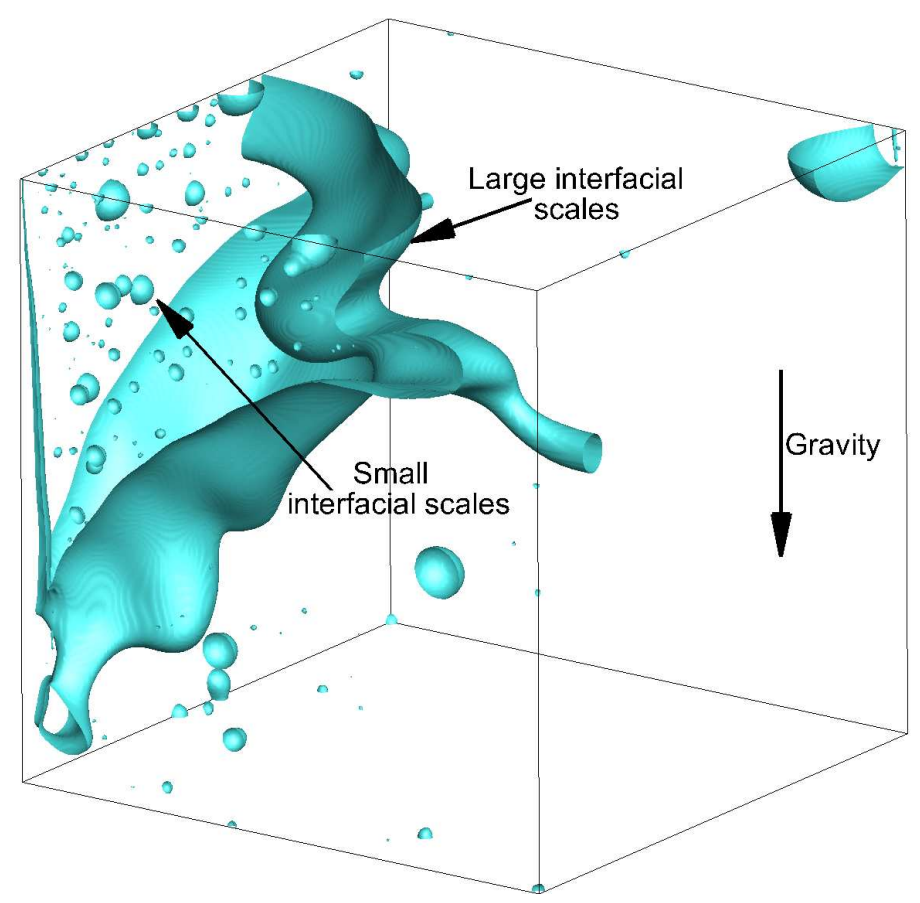

Figure 1: Example of multi-scale interfacial character of phase separation for a liquid-liquid two-phase system. The interface is plotted in blue and the black lines represent the wall of the cubic box. Following $[2,3,11], \mathrm{Re}=7000$ and $\mathrm{We}=11$.

Various models are available in literature to tackle with two-phase flows. For dispersed flows, when a scale separation can be assumed between the car- 
rier fluid and the dispersed phase, i.e. droplets or bubbles, Eulerian-Eulerian or Eulerian-Lagrangian models exist [12] in which the continuous phase, also called the carrier fluid, is treated with the classical fluid mechanics equations and the dispersed phase is taken into account through an Eulerian concentration variable or discrete Lagrangian positions and velocities. Specific source terms are added to the mass and momentum equations in order to represent the interaction effects between both phases. When the characteristic size of the interfacial structures is comparable to the macroscopic size of the problem or to the local mesh size, the previous scale separation models are no more valid.

The present work is concentrated on two-phase flow problems involving a wide range of interfacial structures that can be large and small at the same time, depending on the zone of the flow that is considered. Among others, examples are the primary atomization of a liquid jet or sheet [13-15] or the liquid-liquid phase separation $[2,3,11]$ presented in figure 1 . As a starting point, we consider here multi-scale two-phase flows with separate phases in unsteady and turbulent regime. It is assumed that no scale separation exists between large interfacial structures and small turbulent scales. For large Reynolds numbers, performing a Direct Numerical Simulation (DNS) of the flow, i.e. a simulation with numerical time and space scales that are smaller than the physical characteristics of turbulence and interface, is almost impossible as it would require computer resources not available even on the most powerful parallel computers. As a consequence, for a kind of mesoscopic modeling issue, it has to be assumed that all the interfacial scales will be resolved whereas turbulence will be modeled by means of a Large Eddy Simulation (LES) approach [16]. This way, relatively large grids and time steps could be considered that give access to deterministic and unsteady simulations on meshes coarser than with a DNS. However, two basic points have to be specified to lead our mesoscopic representation of the turbulenceinterface interaction: on one hand, the choice of models that allow the solving of deformable interfaces between non miscible fluids and on the other hand, the LES filtering of these models in the framework of two-phase flows. These two points are the main theme of our work. On a general point of view, one can adress the following remarks in order to build a LES two-phase flow modeling with resolved scale interfaces:

- concerning the modeling of two-phase flows with separated phases and non miscible fluids, the most widely used model in the literature is the single fluid or one-fluid model (OFM) [17-19]. This Eulerian model is based on the use of a regular kernel of control volumes on which the in- 
terface is located by means of an auxiliary Eulerian variable. This variable can be the local Volume fraction of one phase in the other phase, i.e. the Volume Of Fluid (VOF) [20], the signed distance function to the interface, i.e. the Level Set approach [21], or the VOF function resulting from the projection of a Lagrangian tracking of the interface, i.e. the Front Tracking method [22]. In these three approaches, the standard incompressible Navier-Stokes equations are considered, with an additive specific capillary source term that accounts for the normal jump of constraints at the interface. In the VOF and Level Set, an additional advection equation of the interface is solved in an Eulerian way while a Lagrangian interface marker equation is considered with the Front Tracking. The one-fluid model allows the representation of twophase flows with separated fluids and deformable interfaces. Another class of models can be considered to have the same kind of two-phase flow representation. It is called the multi-field approach (MFA) [23-25] or segregated phase modeling [19]. This type of two-phase flow modeling was initially designed for a dispersed phase that is represented through an Eulerian variable such as the local concentration of the dispersed phase in the surrounding carrier fluid. However, this EulerEuler model can be extended to large scale and resolved interfaces by introducing a sharpening equation together with the transport of the dispersed phase concentration [23,25]. This sharpening equation is coupled with mass and momentum equations in order to ensure mass conservation to almost zero computer error. The obtained multi-field model allows simulating large scale interfaces in a similar approach as phase field models [26], with an interface that is given a prescribed thickness of several grid cells. The OFM and MFA will be considered in the present article to discriminate the modeling strategy that is most suited for the simulation of turbulent two-phase flows with large interfaces.

- regarding the LES modeling of turbulence, this is an area of fluid mechanics that has been widely studied and developed for single-phase flows past twenty years $[16,27]$. For cons, the LES of two-phase flows with large scale interfaces remains an understudied area. Among the few existing references include the work on LES formulation for the one fluid model and subsequent a priori LES filtering of DNS simulations for the estimate of new specific two-phase contributions in the mass, momentum and VOF advection equations [2,3,28-32]. Two main conclusions arise from these studies: new subgrid terms have to be modeled when the LES of two-phase flows is undertaken and the magnitude of 
these subgrid terms strongly varies depending on the considered physical configuration so that none of these subgrid terms can be neglected a priori, except perhaps the viscous subgrid term. Concerning the modeling of the two-phase LES subgrid terms, most advanced works have been devoted to the inertial and capillary terms with formulations based on Smagorinsky or deconvolution like approaches [30,33]. None of these LES models have clearly demonstrated that it was possible to perform a LES of resolved scale interfaces with success, except perhaps [30] for the atomization of a fuel jet. In addition, no LES model have been proposed for the viscous, advection or mass conservation subgrid terms.

If one refers to the previous literature review, it can be noted that there is a real need to make a comprehensive and systematic a priori study of the two-phase LES subgrid terms appearing in the one-fluid and even more multifield formalisms. In addition, representative models have to be proposed for all subgrid terms and validated against DNS of turbulent two-phase flows. These two lines of research are the main goal of the present work. The article is structured as follows. In the next section, the OFM and MFA are shortly presented with associated numerical methods. A phase inversion benchmark configuration is also proposed for the LES modeling of two-phase flows with multi-scale interfaces. Section 3 is devoted to a priori LES filtering of OFM and MFA as well as comparison of subgrid term magnitude and classification. LES models for two-phase subgrid terms of OFM and MFA is proposed in section 4 . These models are compared and discussed regarding DNS of phase inversion. Conclusions and perspectives are finally drawn in section 5 .

\section{Modeling and simulation of phase inversion}

\subsection{Phase inversion benchmark}

Various configurations of phase inversion benchmark have previously been proposed and used for a priori LES filtering, comparison of interface tracking methods, characterization of multi-scale interfacial flow and comparison of DNS two-phase flow codes [2-4,11,33,34]. A previous configurations was chosen here for its turbulent regime and large range of interfacial scales. The initial condition of the problem is described in Figure 2. An initial cubic blob of light liquid, referred to as fluid 1, is placed in the bottom part of a cubic box filled with a heavier liquid, referred to as fluid 2. The size of the box is $(H, H, H)$, while the size of the blob of light fluid is $(H / 2, H / 2, H / 2)$, with $H=1 \mathrm{~m}$. All outer walls are considered as free-slip impermeable walls. The 


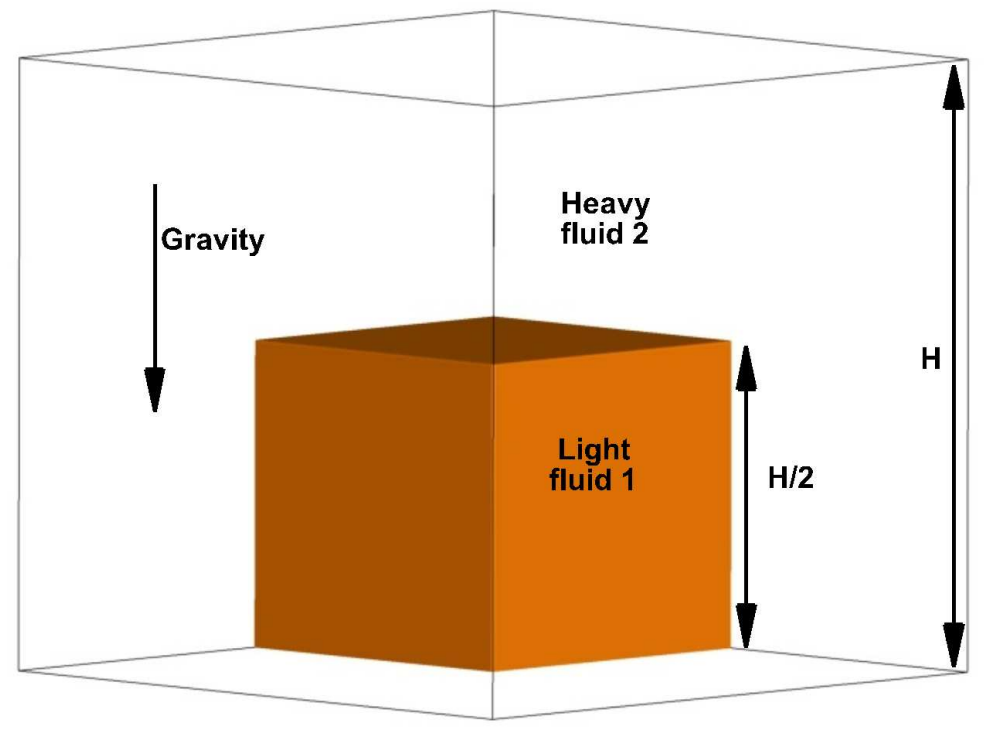

Figure 2: Definition sketch for the phase inversion benchmark in a closed box $[2,3,11]$.

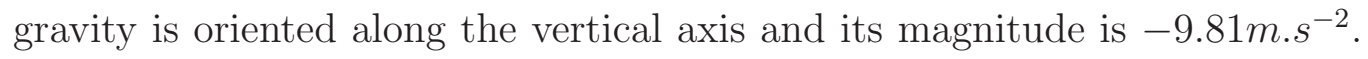
The interest of the present configuration lies in simplicity of initial and final flow configurations, i.e. all light fluid is inside a cubic blob initially and all light fluid is in the top part of the cavity at long times. Another advantage is that phase inversion naturally provides multiple coalescence, break-up and fragmentation events in a kind of atomization process, while not depending on complex initial or forcing conditions such as those encountered in real turbulent two-phase flow configurations [13] [35]. The characteristics of fluid 1 and 2 are $\rho_{1}=900 \mathrm{~kg} \cdot \mathrm{m}^{-3}, \rho_{2}=1000 \mathrm{~kg} \cdot \mathrm{m}^{-3}, \mu_{1}=0.1$ Pa.s and $\mu_{2}=0.001 P a . s$. The constant surface tension coefficient is chosen equal to $0.45 N . m^{-1}$.

The fluids and flow properties that control the phase inversion dynamics can be expressed in terms of Atwood, Reynolds and Weber numbers. These 

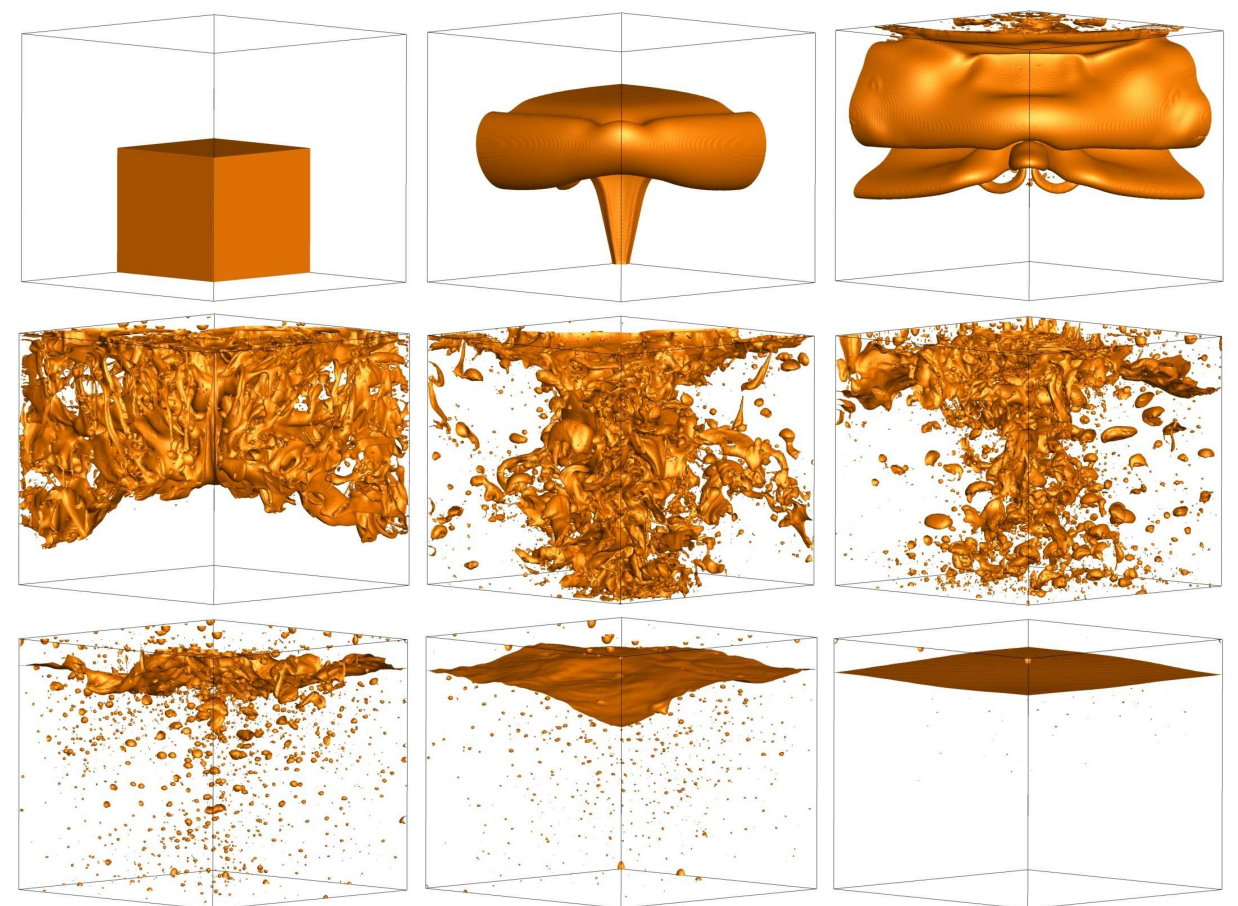

Figure 3: Simulation of a phase inversion in a closed box with a one-fluid model - The interface between the two liquids is plotted in orange - from left to right and top to bottom, the dimensionless time is $0,0.246,0.492,0.738$, $0.984,1.329,1.969,3.396$ and 15.305 .

numbers are defined as

$$
\begin{aligned}
A t & =\frac{\rho_{2}-\rho_{1}}{\rho_{1}+\rho_{2}}, \\
R e_{1}=\frac{\rho_{1} H U_{g}}{\mu_{1}} \text { and } R e_{2} & =\frac{\rho_{2} H U_{g}}{\mu_{2}}, \\
W e_{1}=\frac{\rho_{1} H U_{g}^{2}}{\sigma} \text { and } W e_{2} & =\frac{\rho_{2} H U_{g}^{2}}{\sigma},
\end{aligned}
$$

where the gravitational velocity $U_{g}$ is obtained as the balance between the pressure forces on the light fluid and the net gravity force on this fluid $[3,4]$. It reads

$$
U_{g}=\frac{\rho_{2}-\rho_{1}}{\rho_{1}} \sqrt{\frac{g H}{2}}=A t^{1 / 2} \frac{\rho_{1}+\rho_{2}}{\rho_{1}} \sqrt{\frac{g H}{2}}
$$

Based on $U_{g}$ and a characteristic space scale of $H / 2$, the characteristic time 
scale is

$$
t_{c}=\frac{H}{2 U_{g}}=A t^{-1 / 2} \frac{\rho_{1}}{\left(\rho_{1}+\rho_{2}\right)} \sqrt{\frac{H}{2 g}} .
$$

The characteristic parameters of phase inversion are At $=0.053, U_{g}=$ $0.246 \mathrm{~m} . \mathrm{s}^{-1}, R e_{1}=2.214 \cdot 10^{3}, R e_{2}=2.460 \cdot 10^{5}, W e_{1}=1.210 \cdot 10^{2}, W e_{2}=$ $1.345 \cdot 10^{2}$, and $t_{c}=2.032 \mathrm{~s}$. An example of simulation with these parameters is illustrated in figure 3 for a $512^{3}$ grid with a one-fluid model. Due to large Reynolds and Weber numbers, the flow is first dominated by inertial effects induced initially by gravity forces with negligible capillary forces. For larger times, inertia decreases and surface tension forces become predominant. It is observed that initially the light fluid blob goes to the top of the cavity while being subjected to large interface deformation with rupture of ligaments into droplets. In a second step, a kind of atomization process occurs with the phase separation being active inducing coalescence of many droplets with the top layer of light fluid.

\subsection{One-fluid model (OFM)}

\subsubsection{A single velocity formulation for two-phase flows}

As is now well established, incompressible two-phase flows involving fluidfluid interfaces and Newtonian fluids can be modeled by a single set of incompressible Navier-Stokes equations with variable density and viscosity and possibly extra interfacial forces (e.g. the capillary force), together with the transport equation of the phase function $C$. The resulting model takes implicitly into account the mass and momentum jump relations at the interface [36] [18], whereas the continuity of the fluid-fluid and fluid-solid interfaces are taken into account by the $C$ equation. The entire set of equations reads:

$$
\begin{array}{r}
\nabla \cdot \mathbf{u}=0 \\
\rho\left(\frac{\partial \mathbf{u}}{\partial t}+\mathbf{u} \cdot \nabla \mathbf{u}\right)=-\nabla p+\rho \mathbf{g}+\nabla \cdot\left[\mu\left(\nabla \mathbf{u}+\nabla^{t} \mathbf{u}\right)\right]+\mathbf{F}_{s t} \\
\frac{\partial C}{\partial t}+\mathbf{u} \cdot \nabla C=0
\end{array}
$$

where $p$ is the pressure, $\mathbf{F}_{s t}$ is the interfacial force per unit volume and $\rho$ and $\mu$ are the local density and viscosity of the two-phase medium, respectively. In the rest of the article, the viscous stress tensor will be referred to as $\mathbf{S}=\frac{1}{2}\left(\nabla \mathbf{u}+\nabla^{t} \mathbf{u}\right)$. Motion of the interface in a given velocity field $\mathbf{u}$ is considered in equation (8) through the $\mathrm{VOF}$ function $C$. In the present 
work, it is chosen instead to use the signed distance function to the interface $\phi$, that satisfies the following equation:

$$
\frac{\partial \phi}{\partial t}+\mathbf{u} \cdot \nabla \phi=0
$$

Capillary effects are inserted in the source term $\mathbf{F}_{s t}$ in the form $\mathbf{F}_{s t}=$ $\sigma \kappa \mathbf{n}_{i} \delta_{i}$, where $\sigma$ denotes the surface tension, $\kappa$ is the local mean curvature of the interface, i.e. $\kappa=-\nabla \cdot \mathbf{n}=-\nabla \cdot\left(\frac{\nabla C}{\|\nabla C\|}\right), \mathbf{n}_{i}$ is the unit vector normal to the interface and $\delta_{i}$ is the interface Dirac function [37], such that $\mathbf{n}_{i} \delta_{i}=\nabla C$.

The above OFM is almost identical to the classical incompressible NavierStokes equations, except that it involves an extra interfacial force and the local properties $\rho$ and $\mu$ of the equivalent fluid depend on $C$, as does the capillary force. Localizing the interface requires solving the additional advection equation for the Level Set $\phi$ such that $\phi=0$ at the interface and $\phi>0$ (resp. $<0$ ) in fluid 2 (resp. 1). In this case, $C$ is defined as the Heaviside function $C=H(\phi)[21]$. It is assumed that all space and time scales present in the flow are resolved, so that no turbulence model is required.

\subsubsection{Numerical methods}

The in-house computational fluid dynamics library developed at ONERA called DyJeAT (Dynamic of Jet ATomization) is used. Velocity/pressure coupling is ensured with classical projection methods [38] [39] on a staggered grid. The Ghost Fluid method $[21,40]$ is implemented to deal with surface tension forces as well as density and viscosity jumps. Instead of inserting a surface tension volume force in the momentum equations as was proposed by [37], with the Ghost fluid method, the jump relations are directly used in prediction and projection steps so as to handle density and viscosity contrasts at the interface as well as capillary effects. In the LES formalism, the delta formulation of [37] is kept only to provide an interpretation of the under resolved capillary forces. The Level Set approach is also used for tracking interfaces, as previously specified. To avoid singularities in the $\phi$ field, the fifth order conservative WENO [41] scheme is applied to discretize space advection terms together with a $3^{\text {rd }}$ order Runge-Kutta TVD scheme for time derivatives [42]. When the Level Set advection is solved, high velocity gradients can generate artificial spreading or stretching of $\phi$ which then no longer remains a distance function. A redistancing algorithm [43] is thus considered to restore the distance property of $\phi$, i.e. $\|\nabla \phi\|=1$. Details of 
implementation and validation can be found in $[15,44,45]$.

\subsection{Multi-field approach (MFA)}

\subsubsection{A two-velocity model for two-phase flows}

The two-fluid model of Ishii [46] is used for a two-phase flow. Initially, the Ishii Eulerian model for two-phase flows was considering the interaction between a carrier fluid (field 1) and a dispersed phase (field 2) under scale separation assumption. In our approach, the density, the viscosity and the local velocity are defined for each field as for Ishii model. However, field $k=1$ corresponds to the first fluid and $k=2$ to another fluid that is immiscible inside field 1. This study is restricted to incompressible and isothermal cases with a constant density in each field. The following governing equations are solved for each field $k$ :

$$
\begin{array}{r}
\frac{\partial \alpha_{k} \rho_{k}}{\partial t}+\nabla \cdot\left(\alpha_{k} \mathbf{u}_{k} \rho_{k}\right)=0, \\
\frac{\partial \alpha_{k} \rho_{k} \mathbf{u}_{k}}{\partial t}+\nabla \cdot\left(\alpha_{k} \rho_{k} \mathbf{u}_{k} \otimes \mathbf{u}_{k}\right)=-\alpha_{k} \nabla p_{k} \\
+\alpha_{k} \rho_{k} \mathbf{g}+\nabla \cdot\left[\alpha_{k} \mu_{k} \mathbf{S}_{k}\right]+\mathbf{F}_{s t, k}+\mathbf{F}_{d, k},
\end{array}
$$

With $\alpha_{k}$ the local volume fraction of field $k, \mathbf{S}_{k}$ the viscous stress tensor of field $k$, i.e. $S_{i j, k}=\frac{\partial u_{i, k}}{\partial x_{j, k}}+\frac{\partial u_{j, k}}{\partial x_{i}}-\frac{2}{3} \frac{\partial u_{i, k}}{\partial x_{i}} \delta_{i j}$ and $\mathbf{F}_{d, k}$ a volume force ensuring the coupling between the continuous fields through a drag force. This drag force is crucial since it allows coupling the velocity of the two continuous fields at the interface. Thus, a new drag force expression has been developed to deal with large interfaces within the MFA. Details can be found in $[25,47]$. The modeling of large interfaces and surface tension forces with Ishii model is based on a volumetric formulation [23, 25, 48] taken from [37]. It reads $\mathbf{F}_{s t, k}=\alpha_{k} \sigma \kappa_{k} \mathbf{n}_{i, k} \delta_{i}$ with $\kappa_{k}=-\nabla \cdot \mathbf{n}_{k}=-\nabla \cdot\left(\frac{\nabla \alpha_{k}}{\left\|\nabla \alpha_{k}\right\|}\right)$ and $\mathbf{n}_{i, k} \delta_{i}=\nabla \alpha_{k}$.

\subsubsection{Numerical methods}

The code $N E P T U N E \_C F D$ is based on an Eulerian approach with a finite volume discretization. In the code $N E P T U N E \_C F D$, the assumption of a common pressure for all fields is made. The solver SIMPLE (Semi-Implicit Method for Pressure-Linked Equations) is used [49] with a collocated arrangement for all variables. An iterative coupling of the equations is implemented to ensure mass conservation. Details are given in $[25,50]$. 
By nature, the Ishii model is diffuse as it represents the interaction between two phases (or fields) through a mixed Eulerian representation of the different fluids in a given volume. Here, the idea is to tackle with sharp interfaces, even if the MFA is a priori not adapted to a discontinuous representation of a two-phase flow. In order to limit the interface smearing induced by the resolution of the two-fluid equations, an interface sharpening equation, initially proposed by [51] and adapted to the two-fluid formulation, is solved for each continuous field [50]:

$$
\frac{\partial \alpha_{k}}{\partial t}+\nabla \cdot\left(\alpha_{k}\left[1-\alpha_{k}\right] \mathbf{n}_{i, k}\right)=\epsilon \nabla^{2} \alpha_{k}
$$

With $\mathbf{n}_{i, k}$ the interface normal vector pointing outside phase $k$. The value of $\epsilon$ is chosen equal to half the space step $\Delta x$ while the time step of the interface sharpening equation is taken equal to $\Delta x / 32$. With these parameters, the obtained final interface thickness is always equal to 5 cells whatever the initial interface diffusion $[23,52]$.

\section{Model comparison on macroscopic quanti- ties}

Several macroscopic quantities characterizing the evolution of the flow field are of primary interest to check whether the two models describe the same two-phase motion. These quantities can be expected to be a priori essentially independent of the choice of the model and interface tracking techniques used in the computations. In all of what follows, we use the characteristic or color function $C$ that is defined with respect to fluid 2 , so that $C=1$ in fluid 2 and $C=0$ in fluid 1. Among these physically relevant quantities and in agreement with previous work on the phase inversion benchmark $[3,4]$, we select:

- the domain integrals of potential energies $E_{p, 2}=\int_{\Omega} C \rho_{2} g y d V$ and $E_{p, 1}=\int_{\Omega}(1-C) \rho_{1} g y d V$ in fluid 1 and 2 (with $g=\|\mathbf{g}\|$ ) and the domain integrals of kinetic energies $E_{k, 2}=\frac{1}{2} \int_{\Omega} C \rho_{2} \mathbf{u}^{2} d V$ and $E_{k, 1}=\frac{1}{2} \int_{\Omega}(1-C) \rho_{1} \mathbf{u}^{2} d V$ will also be stored at each time step. They will help monitoring the conversion of potential energy into kinetic energy. they provide a sort of characterization of the stratification inside the fluids. Due to the simple topology of the interface in the initial and final stages, it can be shown that $E_{p, 1}=\rho_{1} g \frac{H^{4}}{32}$ and $E_{p, 2}=\rho_{2} g \frac{31 H^{4}}{32}$ for $t=0$ and $E_{p, 1}=\rho_{1} g \frac{15 H^{4}}{128}$ and $E_{p, 2}=\rho_{2} g \frac{49 H^{4}}{128}$ for $t \rightarrow \infty$. The typical magnitude of the kinetic energy in both fluids may be estimated 
by using the gravitational velocity $U_{g}$, yielding $E_{k, 1}=O\left(\frac{1}{16} \rho_{1} H^{3} U_{g}^{2}\right)$ and $E_{k, 2}=O\left(\frac{1}{16} \rho_{2} H^{3} U_{g}^{2}\right)$.

- the time evolution of the volume integral of the enstrophy in both fluids will also be recorded. This quantity is defined as $E_{r, 1}=\frac{1}{2} \int_{\Omega}(1-C) \mathbf{w}^{2} d V$ and $E_{r, 2}=\frac{1}{2} \int_{\Omega} C \mathbf{w}^{2} d V$, respectively, $\mathbf{w}=\nabla \times \mathbf{u}$ denoting the vorticity.

The simulated values are made dimensionless by using the previous reference estimates of time as well as potential and kinetic energies. For enstrophy, the time maximum of the value obtained with the one fluid model is used, as was proposed in [4]. A synthesis of the dimensionless parameters is given in table 1 . The results are presented in figure 4. For potential and kinetic energies in fluid 1 and 2, it is observed that the two models are in rather good agreement. They capture the same time evolution with peak of energies being located at the same instants. For intermediate times, when droplet generation is maximum, some discrepancies are noticed. They are due to the different interface tracking techniques and also to single velocity field representation of the two-phase flow by the OFM, compared to twovelocity description brought by the MFA. Concerning enstrophy in fluid 1 and 2, the OFM brings two to three times larger magnitudes than MFA. The peak of enstrophy is located at the same dimensionless time for both models, i.e. $t^{*} \approx 2.5$ in fluid 1 and $t^{*} \approx 4$ in fluid 2 . The major differences observed concerning enstrophy were also reported in [4] concerning the comparison of different codes on different grids (up to $2048^{3}$ mesh). It was demonstrated that on a vorticity point of view, a $512^{3}$ grid is not enough to perform a real DNS for the phase inversion benchmark whereas potential and kinetic energies can be assumed converged. In the rest of the work, it will be considered that a $512^{3}$ grid simulation is enough for performing a DNS of the phase separation benchmark and provide a representative simulation of this complex two-phase flow, illustrated in figure 3. If different information is not specified, all the results presented in the following sections are investigated with the OFM and MFA on a $512^{3}$ regular Cartesian mesh.

\section{A priori filtering of turbulent two-phase flows}

The Large Eddy Simulation filtering of motion equation for two-phase flows is now explained. Thanks to the phase function $C$ indicating phase $k$, a lowpass frequency filtering operator $G$ defining the spatial filtering of a given variable $\Psi$ is written as the convolution product of $G$ with $\Psi$ as $\bar{\Psi}=G o \Psi$. The phase-weighted filtering of the velocity $\mathbf{u}$, i.e. called Favre average, is 


\begin{tabular}{ccc}
\hline Parameter & Value & Unit \\
\hline$t^{*}=\frac{t}{t_{c}}=\frac{t U_{g}}{H}=\frac{t\left(\rho_{2}-\rho_{1}\right)}{\rho_{1}} \sqrt{\frac{g}{2 H}}$ & $\frac{t}{2.032}$ & - \\
$\frac{15 \rho_{1} g H^{4}}{128}$ the potential energy in fluid 1 for $t \rightarrow \infty$ & 1035 & $\mathrm{~J}$ \\
$\frac{49 \rho_{2} g H^{4}}{128}$ the potential energy in fluid 2 for $t \rightarrow \infty$ & 3755 & $\mathrm{~J}$ \\
$E_{\text {cin }, 1}^{*}=\frac{E_{\text {cin }, 1}}{1 / 16 \rho_{1} U_{g}^{2} H^{3}}$ & $\frac{E_{c i n, 1}}{3.4063}$ & - \\
$E_{\text {cin }, 2}^{*}=\frac{E_{c i n, 2}}{1 / 16 \rho_{2} U_{g}^{2} H^{3}}$ & $\frac{E_{c i n, 2}}{3.7847}$ & - \\
Maximum of enstrophy in fluid 1 (one-fluid model) & 56.72 & $\mathrm{~m}^{3} \cdot \mathrm{s}^{-2}$ \\
Maximum of enstrophy in fluid 2 (one-fluid model) & 224.03 & $\mathrm{~m}^{3} \cdot \mathrm{s}^{-2}$ \\
\hline
\end{tabular}

Table 1: Parameters used to define the dimensionless variables for phase inversion benchmark.

also defined as $\tilde{\mathbf{u}}=\frac{\overline{\rho \mathbf{u}}}{\bar{\rho}}$ for the OFM whereas it reads $\tilde{\mathbf{u}}_{k}=\frac{\overline{\alpha_{k} \mathbf{u}_{k}}}{\overline{\alpha_{k}}}$ for the MFA. It is assumed here that the commutation between the spatial filter and the derivative is satisfied, as in single-phase LES modeling [16,53]. This assumption is reasonable as the commutation error depends more on the topology of the mesh than on the characteristic of the flow $[54,55]$. In our simulation, the mesh is regular and isotropic so that commutation errors should be negligible.

By applying the filtering operators to the one fluid and multi-field models, specific subgrid terms appear in the LES formalism of two-phase flow with resolved scale interfaces. These subgrid terms have previously been discussed in $[3,28,31,32,56]$ for the OFM and in [57] for the MFA. These two-phase LES terms are associated respectively to acceleration, inertia, viscous effects and interface presence. Their presence is due to the filtering and averaging operations of the mass and momentum equations. The common terms for the OFM and MFA are presented in table 2. The formulations are written as they should be estimated by a priori filtering. In fact, for the sake of simplicity, for all $\overline{\nabla \Psi}$ like terms, the commutation between the filtering and derivation operations has been assumed, such that it has been considered that for any variable $\Psi, \overline{\nabla \Psi}=\nabla \bar{\Psi}$. For OFM, the filtered curvature of the interface $\hat{\kappa}$ is equal to $-\nabla \cdot\left(\frac{\overline{\nabla C}}{\|\overline{\nabla C}\|}\right)$ according to the filtering of Brackbill CSF force [37]. An equivalent expression is used for the filtered curvature 

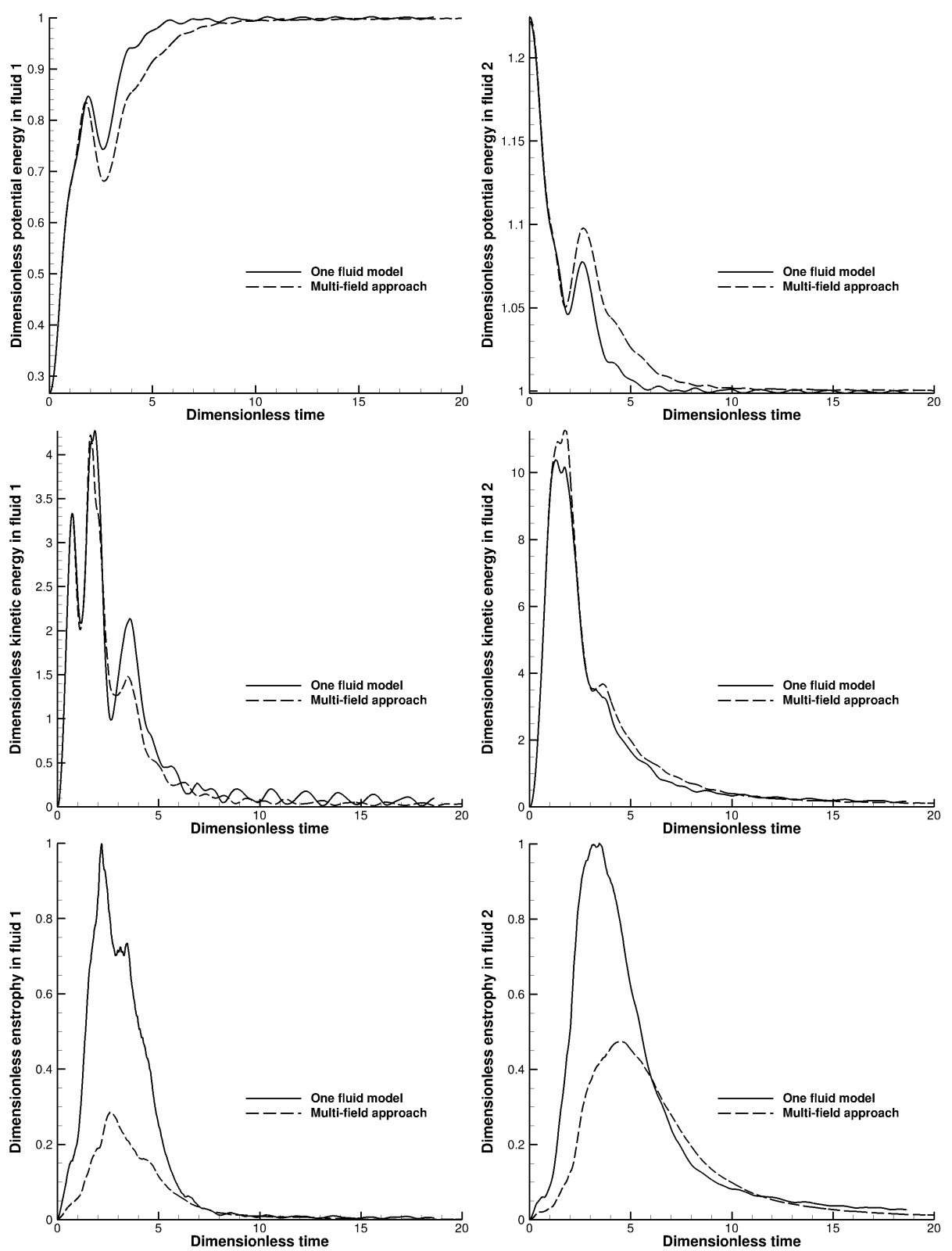

Figure 4: Time evolution of macroscopic quantities for one-fluid and multifield models.

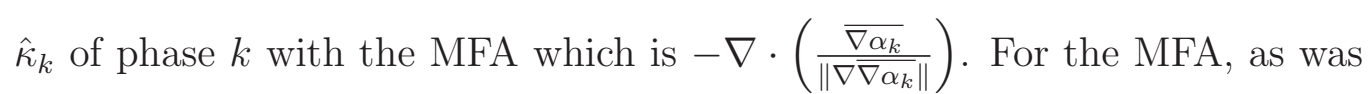
demonstrated by [57], two others subgrid terms exist associated to pressure gradient and drag force. They are not considered in the present work as they do not exist in the OFM. For more details, the reader is referred to [57]. 


\begin{tabular}{|c|c|c|}
\hline Subgrid term & Filter ${ }^{-}$ & Favre average * \\
\hline \multicolumn{3}{|l|}{ One-fluid model } \\
\hline Time derivative $\tau_{t}$ & $\rho \frac{\partial \mathbf{u}}{\partial t}-\bar{\rho} \frac{\partial \mathbf{u}}{\partial t}$ & $\left(\rho_{2}-\rho_{1}\right) \tilde{\mathbf{u}} \tau_{i a}$ \\
\hline Convection $\tau_{c}$ & $\overline{\rho \mathbf{u} \cdot \nabla \mathbf{u}}-\bar{\rho} \overline{\mathbf{u}} \cdot \overline{\nabla \mathbf{u}}$ & $\overline{\rho \mathbf{u} \cdot \nabla \mathbf{u}}-\bar{\rho} \tilde{\mathbf{u}} \cdot \nabla \tilde{\mathbf{u}}$ \\
\hline Diffusion $\tau_{d}$ & $\overline{\nabla \cdot[2 \mu \mathbf{S}]}-\nabla \cdot[2 \bar{\mu} \overline{\mathbf{S}}]$ & $\overline{\nabla \cdot[2 \mu \mathbf{S}]}-\nabla \cdot \mid 2 \bar{\mu} \tilde{\mathbf{S}}$ \\
\hline Surface tension $\tau_{s t}$ & $\sigma(\overline{\kappa \nabla C}-\hat{\kappa} \overline{\nabla C})$ & $\sigma(\overline{\kappa \nabla C}-\hat{\kappa} \overline{\nabla C})$ \\
\hline Interface advection $\tau_{i a}$ & $\overline{\mathbf{u} \cdot \nabla C}-\overline{\mathbf{u}} \cdot \overline{\nabla C}$ & $\overline{\mathbf{u} \cdot \nabla C}-\tilde{\mathbf{u}} \cdot \overline{\nabla C}$ \\
\hline \multicolumn{3}{|l|}{ Multi-field model } \\
\hline Time derivative $\tau_{t}$ & $\rho_{k}\left(\frac{\overline{\partial \alpha_{k} \mathbf{u}_{k}}}{\partial t}-\frac{\partial \overline{\alpha_{k}} \overline{\mathbf{u}}_{k}}{\partial t}\right)$ & - \\
\hline Convection $\tau_{c}$ & $\begin{array}{r}\rho_{k}\left(\overline{\nabla \cdot\left(\alpha_{k} \mathbf{u}_{k} \otimes \mathbf{u}_{k}\right)}\right. \\
\left.-\nabla \cdot\left(\bar{\alpha}_{k} \overline{\mathbf{u}}_{k} \otimes \overline{\mathbf{u}}_{k}\right)\right)\end{array}$ & $\begin{array}{c}\rho_{k}\left(\overline{\nabla \cdot\left(\alpha_{k} \mathbf{u}_{k} \otimes \mathbf{u}_{k}\right)}\right. \\
\left.-\nabla \cdot\left(\bar{\alpha}_{k} \tilde{\mathbf{u}}_{k} \otimes \tilde{\mathbf{u}}_{k}\right)\right)\end{array}$ \\
\hline Diffusion $\tau_{d}$ & $\begin{array}{r}\mu_{k}\left(\overline{\nabla \cdot\left[\alpha_{k} \mathbf{S}_{k}\right]}\right. \\
\left.-\nabla \cdot\left[\bar{\alpha}_{k} \overline{\mathbf{S}}_{k}\right]\right)\end{array}$ & - \\
\hline Surface tension $\tau_{s t}$ & $\begin{array}{r}\sigma\left(\overline{\alpha_{k} \kappa_{k} \nabla \alpha_{k}}\right. \\
\left.-\overline{\alpha_{k}} \hat{\kappa}_{k} \overline{\nabla \alpha_{k}}\right)\end{array}$ & $\begin{array}{l}\sigma\left(\overline{\alpha_{k} \kappa_{k} \nabla \alpha_{k}}\right. \\
\left.-\overline{\alpha_{k}} \hat{\kappa}_{k} \overline{\nabla \alpha_{k}}\right)\end{array}$ \\
\hline Interface advection $\tau_{i a}$ & $\rho_{k}\left(\overline{\nabla \cdot\left(\alpha_{k} \mathbf{u}_{k}\right)}-\nabla \cdot\left(\overline{\alpha_{k}} \overline{\mathbf{u}}_{k}\right)\right)$ & - \\
\hline
\end{tabular}

Table 2: Two-phase LES subgrid terms obtained with one-fluid and multifield models by $A$ priori filtering.

It can be observed that 5 subgrid LES terms exist with the standard filter whereas less terms appear with a Favre average, i.e. 4 for the OFM and 2 for the MFA. It has to be kept in mind that the subgrid terms $\tau_{c}, \tau_{d}, \tau_{s t}$ and $\tau_{i a}$ appear in the mass, momentum and interface tracking equations as extra terms, as soon as a real LES simulation is investigated. For example, if $\tau_{i a}$ is not zero, the flow is no more divergence free in the cells cut by the interface, even if no phase change is considered. In the present work, the time derivative subgrid term $\tau_{t}$ has not been considered. Indeed, its a priori filtering would require to store at least two successive fields of the $512^{3}$ DNS simulations. This was not achieved due to its numerical cost.

The first interesting issue is the estimate of the relative order of magnitude of the specific two-phase subgrid LES terms which appear in mass, momentum and interface advection equations. An a priori filtering of the DNS results have been provided for $\tau_{c}, \tau_{d}, \tau_{s t}$ and $\tau_{i a}$ at the enstrophy peak, 


\begin{tabular}{ccc}
\hline \hline Subgrid term & Filter ${ }^{-}$ & Favre average $\tilde{*}$ \\
\hline \hline One-fluid model & & \\
\hline$\tau_{c}$ & 7.54 & 3.01 \\
$\tau_{d}$ & 1.19 & 0.14 \\
$\tau_{s t}$ & 37.81 & 37.81 \\
$\tau_{i a}$ & 10.29 & 9.61 \\
\hline Multi-field model & & \\
\hline$\tau_{c}$ & 2.24 & 0.86 \\
$\tau_{d}$ & 0.14 & - \\
$\tau_{s t}$ & 5.29 & 5.29 \\
$\tau_{i a}$ & 1.07 & - \\
\hline \hline
\end{tabular}

Table 3: Relative magnitude (in \%) of the vertical component of two-phase LES subgrid terms obtained with one-fluid and multi-field models after $a$ priori filtering. The terms are made dimensionless by the magnitude of the vertical component of the largest resolved DNS term, i.e. the convective contribution. The width of the low-pass filtering operator $G$ is 2 cells in each direction.

i.e. for $t^{*} \approx 3$. The averaged magnitude of the subgrid terms is normalized by the maximum, at each time step, of the filtered resolved contributions of the flow (inertia for the momentum equation and advection for the phase function equation). The width of the low-pass filtering operator $G$ is 2 cells in each direction. From a global point of view, the magnitude of the vertical component of the subgrid terms is larger than the contributions in the $\mathrm{x}$ and $y$ directions. In the z-direction or gravity direction, the flow is strongly anisotropic. This is induced by the key phenomenon of the phase separation, i.e. the buoyancy force. Moreover, it was observed that the magnitude of the subgrid terms is almost the same in the $\mathrm{x}$ and $\mathrm{y}$ directions. In the rest of this section, only the vertical component of the subgrid terms will be considered. According to table 3, a classification of LES subgrid terms can be deduced:

- contrary to what could be intuitively expected, the inertial or convective subgrid term $\tau_{c}$ is not the predominant term. With the OFM, its averaged magnitude is $7.5 \%$ the filtered resolved convective term with the top hat filtering and $3 \%$ with the Favre average. Concerning the MFA, the averaged estimated values of the subgrid convective term are $2.2 \%$ with filter - and $0.9 \%$ with the Favre average. This term cannot be neglected in LES modeling of resolved scale two-phase flows. For both OFM and MFA, using 


\begin{tabular}{ccc}
\hline \hline Subgrid term & Filter ${ }^{-}$with 2 cells width & Filter ${ }^{-}$with 4 cells width \\
\hline \hline$\tau_{c}$ & 7.54 & 13.24 \\
$\tau_{d}$ & 1.19 & 1.96 \\
$\tau_{s t}$ & 37.81 & 44.18 \\
$\tau_{i a}$ & 10.29 & 16.17 \\
\hline \hline
\end{tabular}

Table 4: Effect of width of the low-pass filtering operator $G$ on the relative magnitude (in \%) of the vertical component of two-phase LES subgrid terms obtained with one-fluid model after a priori filtering. The terms are made dimensionless by the magnitude of the vertical component of the largest resolved DNS term, i.e. the convective contribution.

a Favre average reduces the order of magnitude of the subgrid contribution by a factor of 2 .

- concerning the subgrid viscous contribution $\tau_{d}$, it is clearly the lower one, compared to convective, surface tension and interface advection, with both the OFM and MFA modeling approaches. This conclusion was previously given by $[2,3,29,31]$ for various physical configurations. Its order of magnitude is two-times less than the filtered resolved convective terms. This term could be discarded in future LES modeling of two-phase flows.

- the capillary subgrid contribution $\tau_{s t}$ is larger than $\tau_{c}, \tau_{d}$ and $\tau_{i a}$. This conclusion is different from what was obtained for example by [32] for an isolated bubble interacting with a Homogeneous Isotropic Turbulence. This is mainly due to the fact that in our phase separation problem, a kind of atomization process is observed for $t^{*}=3$. In our configuration, many droplets are generated. They are poorly resolved on the $512^{3}$ grid with only a few grid cells (3 to 6 ) over a droplet diameter. This resolution effect is measured by the magnitude of $\tau_{s t}$. Indeed, as soon as the poorly resolved interfacial structures are filtered, the corresponding subgrid terms associated to interface, i.e. the surface tension contribution here, becomes significant compared to the filtered convective DNS terms. The same behavior arises for OFM and MFA. As a conclusion, for dispersed flows without scale separation, the subgrid surface tension LES term cannot be neglected. This is the predominant filtered effect. Contrary to what was noticed for the subgrid convective contribution, using a Favre average does not allow to decrease the magnitude of $\tau_{s t}$. This term is highly depend on the interfacial structure and the associated fluid properties. The Favre average uses the density in the filtering 


\begin{tabular}{cccc}
\hline \hline Subgrid term & $128^{3}$ DNS mesh & $256^{3}$ DNS mesh & $512^{3}$ DNS mesh \\
\hline \hline Filter ${ }^{-}$ & & & \\
\hline$\tau_{c}$ & 14.73 & 10.03 & 7.54 \\
$\tau_{d}$ & 0.68 & 0.75 & 1.19 \\
$\tau_{s t}$ & 16.72 & 19.54 & 37.81 \\
$\tau_{i a}$ & 23.43 & 16.60 & 10.29 \\
\hline Favre average ${ }^{*}$ & & & \\
\hline$\tau_{c}$ & 8.99 & 4.49 & 3.01 \\
$\tau_{d}$ & 0.18 & 0.14 & 0.14 \\
$\tau_{s t}$ & 16.72 & 19.54 & 37.81 \\
$\tau_{i a}$ & 22.41 & 15.43 & 9.41 \\
\hline \hline
\end{tabular}

Table 5: Effect of the DNS mesh size on the relative magnitude (in \%) of the vertical component of two-phase LES subgrid terms obtained with onefluid model after a priori filtering. The terms are made dimensionless by the magnitude of the vertical component of the largest resolved DNS term, i.e. the convective contribution. The width of the low-pass filtering operator $G$ is 2 cells in each direction.

operations. In the present work, no particular attention has been paid to the local anisotropy of the interface. This way, when a given quantity is filtered in a cell cut by the interface, the density used can be $\rho_{1}, \rho_{2}$ or an isotropic average of the two. It has been demonstrated by [58] that for resolved scale two-phase flows, anisotropic filters should be used in order to build correct LES models in an heterogeneous medium such as the phase separation flow.

- the interface advection subgrid term is more important with OFM than with MFA. The diffuse character of the interface in MFA is certainly responsible for the lower order of magnitude of $\tau_{i a}$ with MFA compared to OFM. As for $\tau_{s t}$, using a Favre average instead of filter ${ }^{-}$does not decrease the magnitude of the interface advection subgrid term. In fact, the Favre average reduces the importance of the subgrid terms as soon as the filtering is applied to the velocity field. This effect does not hold when filtering interface depending terms. The subgrid term $\tau_{i a}$ is clearly not negligible with OFM while it could be discarded with MFA.

On a general point of view, the magnitude of the two-phase LES subgrid terms is lower with MFA than with OFM. This is a direct consequence of the diffuse character of the two-fluid model. In the OFM, every change of 
interfacial position is directly correlated to velocity and volume fraction gradients so that the subgrid terms $\tau_{c}, \tau_{s t}$ and $\tau_{i a}$ are always significative in the phase inversion problem when the fragmentation mechanisms are observed. In table 4 , the width of the low-pass filtering operator $G$ is changed from 2 cells witdh to 4 cells. The effect of this change on the relative magnitude (in \%) of two-phase LES subgrid terms is considered with OFM. As was previously observed in a priori filtering works $[3,31]$, the larger the compact support of the filter, the higher the magnitude of the subgrid terms is. This is particularly noticeable for $\tau_{c}$ and $\tau_{i a}$.

To finish with a priori filtering of DNS results, three simulations are investigated with OFM on $128^{3}, 256^{3}$ and $512^{3}$ grids. Even if with the two coarser grids, we are not really able to perform true "DNS", the main goal here is to estimate the effect of implicit LES, i.e. under resolved DNS, on two-phase subgrid contributions. The results are reported in table 5. With top hat filter ', refining the mesh size reduces the magnitude of the convective and interface advection subgrid terms, whereas the diffusive viscous and surface tension contributions increase. These observations are intuitive for $\tau_{c}$ and $\tau_{i a}$, not for $\tau_{d}$ and $\tau_{s t}$. In fact, the filtering effect on velocity decreases with the local mesh size. This is a classical tendency that is observed in single phase flows. This effect is recovered for two-phase flows and resolved scale interfaces as in the present phase separation problem, the flow is isothermal without phase change and so the velocity field is continuous across interface. On a kinematic point of view, the problem is continuous and an isotropic filtering is well adapted for LES filtering. On the contrary, when filtered volume fraction terms are put into play, such as in $\tau_{s t}$, the behavior of the subgrid term magnitude according to grid refinement is totally different. Indeed, the more you refine, the more small structures you resolve. As a consequence, the magnitude of the subgrid term is increased as more interfacial structures of larger curvature are obtained. In this case, using a phase-conditioned filter [58] should perhaps be more suited to decrease the filtering effect when refining the simulation mesh. Concerning the viscous term, it can be assumed almost constant over all grids. A clear explanation is not straightforward. The same tendencies are observed all subgrid terms with the Favre average. The same conclusions hold. 


\section{LES modeling of turbulent two-phase flows \\ 5.1 Large Eddy Simulation (LES) models for two-phase flows involving resolved scale interfaces}

\begin{tabular}{|c|c|c|c|c|c|}
\hline Physical case & $\overline{\tau_{t}}$ & $\tau_{c}$ & $\tau_{d}$ & $\tau_{s t}$ & $\tau_{i a}$ \\
\hline $\begin{array}{c}\text { THI interacting } \\
\text { with a } 2 \mathrm{D} \text { droplet } \\
{[31]}\end{array}$ & - & $\begin{array}{c}\text { Smagorinsky }[5] \\
\text { Wale }[6], \text { Bardina }[7] \\
\text { Mixed [8] }\end{array}$ & - & - & - \\
\hline $\begin{array}{c}\text { THI interacting } \\
\text { with a 3D droplet } \\
{[32]}\end{array}$ & $\begin{array}{c}\text { Bardina } \\
{[7]}\end{array}$ & $\begin{array}{c}\text { Bardina } \\
{[7]}\end{array}$ & - & - & $\begin{array}{c}\text { Bardina } \\
{[7]}\end{array}$ \\
\hline $\begin{array}{c}\text { Liquid jet } \\
\text { atomization } \\
{[30]}\end{array}$ & - & $\begin{array}{l}\text { Smagorinsky } \\
\text { Lilly } \\
{[5]}\end{array}$ & - & - & $\begin{array}{c}\text { Smagorinsky } \\
\text { Lilly } \\
{[5]}\end{array}$ \\
\hline $\begin{array}{c}\text { Phase inversion } \\
\text { in a } 3 \mathrm{D} \text { cavity } \\
{[32]}\end{array}$ & - & - & - & $\begin{array}{c}\text { ADM } \\
{[9]}\end{array}$ & - \\
\hline
\end{tabular}

Table 6: Literature review of LES models that have been investigated for two-phase subgrid LES terms.

The understanding and characterization of the coupling between turbulence and fluid interfaces is a difficult task. As we reported in the introduction section, few experimental $[59,60]$ and numerical works $[2,29,30,33,61-63]$ exists to investigate the formulation of LES models for two-phase flows involving resolved scale interfaces. A synthesis of literature works is proposed in table 6. Among the few existing publications, it can be observed that none of the authors have studied all the subgrid terms for a same two-phase problem. In addition, none of the authors have considered $\tau_{d}$, that is always assumed negligible. The goal of the present section is to contribute, for a given phase inversion benchmark problem, to the evaluation of LES models for all two-phase subgrid terms that have been formulated in section 4, except the time subgrid term $\tau_{t}$.

Based on single-phase LES [16], functional models are first considered for two-phase LES subgrid terms. By nature, these functional models have been designed for inertial (or convective) terms. They rely on an energy cascade assumption and a dissipation modeling of turbulence at small scale. In this 
way, they can only be applied to subgrid terms $\tau_{c}$ and $\tau_{i a}$ as follows:

- the Smagorinsky model [5] is first considered for its simplicity and robust behavior. For the convective term, we classically obtain

$$
\tau_{c}=-2 \nabla \cdot\left(C_{s} \bar{\Delta}\right)^{2}\|\overline{\mathbf{S}}\| \bar{f} \overline{\mathbf{S}}
$$

By analogy, the interfacial subgrid term is written as

$$
\tau_{i a}=-2 \nabla \cdot\left(C_{s} \bar{\Delta}\right)^{2}\|\overline{\mathbf{S}}\| \overline{\nabla C}
$$

With this approach, a subgrid scale LES viscosity can be defined as $\nu_{s}=$ $\left(C_{s} \bar{\Delta}\right)^{2}\|\overline{\mathbf{S}}\|$. The Smagorinsky constant $C_{s}$ is generally chosen between 0.1 and 0.24 .

- The Wall-Adapting Local Eddy-Viscosity (Wale) model [6] which is a modification of the Smagorinsky model in order to better predict turbulence behavior near solid boundaries. In particular, this model recovers the expected asymptotic values of the subgrid viscosity near the wall. It reads

$$
\begin{gathered}
\tau_{c}=-2 \nabla \cdot\left(\nu_{w} \overline{f \mathbf{S}}\right) \\
\tau_{i a}=-2 \nabla \cdot\left(\nu_{w} \overline{\nabla C}\right)
\end{gathered}
$$

with $\nu_{w}=\left(C_{w} \bar{\Delta}\right)^{2} \| \frac{(\overline{\mathbf{Z}}: \overline{\mathbf{Z}})^{3 / 2}}{(\overline{\mathbf{S}}: \overline{\mathbf{S}})^{5 / 2}+(\overline{\mathbf{Z}}: \overline{\mathbf{Z}})^{5 / 4}}$ and $C_{w}$ in the range $0.55-0.6$. In addition,

$$
\overline{\mathbf{Z}}=\overline{S S}+\overline{\Omega \Omega}-\frac{1}{3}(\bar{S}: \bar{S}-\bar{\Omega}: \bar{\Omega}) \overline{\overline{I d}}
$$

with $\Omega$ the rotation tensor defined as

$$
\bar{\Omega}=\frac{1}{2}\left(\nabla \overline{\mathbf{u}}-\nabla^{t} \overline{\mathbf{u}}\right)
$$

In all LES models, $\bar{f}$ is 1 for the OFM whereas $\bar{f}=\overline{\alpha_{k}}$ for MFA. In addition, for this last model, a $k$ index have to be added in all LES expressions for velocity depending terms. To finish with, terms based on $\bar{C}$ have to be switched into terms depending on $\bar{\alpha}_{k}$.

A second class of LES approaches can be used to design models for twophase subgrid terms. It is called structural analysis [16]. The main interest of the structural LES is that it does not rely on an explicit understanding and formulation of the coupling between resolved and filtered scales of turbulence 
(and interfaces in the case of two-phase flows). The objectives and strategy of these approaches are to approximate the inverse of the LES filter and to build models with same mathematical structure as the subgrid term. A very interesting aspect with these formulations is that they use only the resolved filtered variables. Once $G^{-1}$ and the filtered variables are known, the real unknowns can be reconstructed, as well as the subgrid contributions. Two main structural approaches have been selected:

- The Bardina model [7] considers a scale similarity which is to assume that the statistical structure of subgrid terms is similar to the terms evaluated from the smaller resolved scales. With this assumption, the filtering of a product is simply the product of filtered variables, i.e. $\overline{a b}=\bar{a} \bar{b}$. After some manipulations, the two-phase subgrid LES terms read for the OFM:

$$
\begin{array}{r}
\tau_{c}=C_{b}(\overline{\rho \overline{\mathbf{u}} \cdot \nabla \overline{\mathbf{u}}}-\overline{\bar{\rho}} \overline{\overline{\mathbf{u}}} \cdot \overline{\overline{\nabla \mathbf{u}}}) \\
\tau_{d}=C_{b} \overline{\nabla \cdot[2 \bar{\mu} \overline{\mathbf{S}}]}-\nabla \cdot[2 \overline{\bar{\mu}} \overline{\overline{\mathbf{S}}}] \\
\tau_{s t}=C_{b} \sigma(\overline{\bar{\kappa} \overline{\nabla C}}-\overline{\bar{\kappa}} \overline{\overline{\nabla C}}) \\
\tau_{i a}=C_{b} \overline{\overline{\mathbf{u}} \cdot \overline{\nabla C}}-\overline{\overline{\mathbf{u}}} \cdot \overline{\overline{\nabla C}}
\end{array}
$$

with $\overline{\bar{\kappa}}=-\nabla \cdot\left(\frac{\overline{\overline{\nabla C}}}{\|\overline{\overline{\nabla C}}\|}\right)$. For the MFA, it reads:

$$
\begin{array}{r}
\tau_{c}=C_{b} \rho_{k}\left(\overline{\nabla \cdot\left(\bar{\alpha}_{k} \overline{\mathbf{u}}_{k} \otimes \overline{\mathbf{u}}_{k}\right)}-\nabla \cdot\left(\overline{\bar{\alpha}}_{k} \overline{\overline{\mathbf{u}}}_{k} \otimes \overline{\overline{\mathbf{u}}}_{k}\right)\right) \\
\tau_{d}=C_{b} \mu_{k}\left(\overline{\nabla \cdot\left[\bar{\alpha}_{k} \overline{\mathbf{S}}_{k}\right]}-\nabla \cdot\left[\overline{\bar{\alpha}}_{k} \overline{\overline{\mathbf{s}}}_{k}\right]\right) \\
\tau_{s t}=C_{b} \sigma\left(\overline{\bar{\alpha}_{k} \hat{\kappa}_{k} \overline{\nabla \alpha_{k}}}-\overline{\bar{\alpha}}_{k} \overline{\bar{\kappa}}_{k} \overline{\overline{\nabla \alpha_{k}}}\right) \\
\tau_{i a}=C_{b} \rho_{k}\left(\overline{\nabla \cdot\left(\bar{\alpha}_{k} \overline{\mathbf{u}}_{k}\right)}-\nabla \cdot\left(\overline{\bar{\alpha}}_{k} \overline{\overline{\mathbf{u}}}_{k}\right)\right)
\end{array}
$$

with $\overline{\bar{\kappa}}_{k}=-\nabla \cdot\left(\frac{\overline{\overline{\nabla \alpha_{k}}}}{\| \overline{\overline{\nabla \alpha_{k}} \|}}\right)$. The Bardina constant $C_{b}$ is adjustable. A value of 1 has been used here.

- The Approximate Deconvolution Model (ADM) [9] is a general framework for building models that respect the mathematical structure of the subgrid terms. First, an approximation of the inverse $G^{-1}$ is build with a given order of accuracy, in a kind of Taylor expansion. Based on $G^{-1}$, reconstructed unfiltered variables are build and used directly in the native 
structure of the subgrid LES terms as follows for the OFM:

$$
\begin{array}{r}
\tau_{c}=C_{a}\left(\overline{\rho \mathbf{u}^{*} \cdot \nabla \mathbf{u}^{*}}-\bar{\rho} \overline{\mathbf{u}}^{*} \cdot \overline{\nabla \mathbf{u}^{*}}\right) \\
\tau_{d}=C_{a} \overline{\nabla \cdot\left[2 \mu \mathbf{S}^{*}\right]}-\nabla \cdot\left[2 \bar{\mu} \overline{\mathbf{S}^{*}}\right] \\
\tau_{s t}=C_{a} \sigma\left(\overline{\kappa^{*} \nabla C^{*}}-\bar{\kappa}^{*} \overline{\nabla C}\right) \\
\tau_{i a}=C_{a} \overline{\mathbf{u}^{*} \cdot \nabla C^{*}}-\overline{\mathbf{u}}^{*} \cdot \overline{\nabla C^{*}}
\end{array}
$$

And for the MFA:

$$
\begin{array}{r}
\tau_{c}=C_{a} \rho_{k}\left(\overline{\nabla \cdot\left(\alpha_{k}{ }^{*} \mathbf{u}_{k}{ }^{*} \otimes \mathbf{u}_{k}{ }^{*}\right)}-\nabla \cdot\left(\overline{\alpha_{k}{ }^{*} \mathbf{u}_{k}{ }^{*}} \otimes \overline{\mathbf{u}_{k}{ }^{*}}\right)\right) \\
\tau_{d}=C_{a} \mu_{k}\left(\overline{\nabla \cdot\left[\alpha_{k}{ }^{*} \mathbf{S}_{k}^{*}\right]}-\nabla \cdot\left[\overline{\alpha_{k}{ }^{*}} \overline{\mathbf{S}_{k}{ }^{*}}\right]\right) \\
\tau_{s t}=C_{a} \sigma\left(\overline{\alpha_{k}{ }^{*} \kappa_{k}{ }^{*} \nabla \alpha_{k}{ }^{*}}-\overline{\alpha_{k}^{*}} \hat{\left.\kappa_{k}{ }^{*} \nabla \overline{\alpha_{k}{ }^{*}}\right)}\right. \\
\tau_{i a}=C_{a} \rho_{k}\left(\overline{\nabla \cdot\left(\alpha_{k}{ }^{*} \mathbf{u}_{k}{ }^{*}\right)}-\nabla \cdot\left(\overline{\alpha_{k}{ }^{*} \mathbf{u}_{k}{ }^{*}}\right)\right)
\end{array}
$$

with $\mathbf{u}^{*} \approx G_{7}^{-1} o \overline{\mathbf{u}}, C^{*} \approx G_{7}^{-1} o \bar{C}$ and $\alpha_{k}{ }^{*} \approx G_{7}^{-1} o \overline{\alpha_{k}}$. The approximate inverse filter of order $k$ is such that

$$
\begin{array}{r}
G_{k}^{-1}=\sum_{l=0, k}(1-G)^{l} \\
\gamma^{*}=G^{-1} o \bar{\gamma} \approx \sum_{l=0, k}(1-G)^{l} o \bar{\gamma} \approx \bar{\gamma}+(\bar{\gamma}-\overline{\bar{\gamma}})+(\bar{\gamma}-\overline{\bar{\gamma}}+\overline{\bar{\gamma}})+\ldots
\end{array}
$$

We have used a $7^{\text {th }}$ order of approximation for $G^{-1}$, instead of a classical $5^{\text {th }}$ order found in literature $[9,33]$, because we have observed better accuracy of modeled subgrid terms compared to filtered DNS terms. For higher orders, no significative improvements have been found. These results are presented in the next section. It has to be stressed that the Bardina model is no more than the ADM model of $2^{\text {nd }}$ order.

The last type of model that has been considered is the mixed SmagorinskyBardina model [8]. It combines the positivity property of the functional model with the structural representativeness of the Bardina model. Due to the intrinsic construction of the functional part of the model, it can only be applied to convective and interfacial subgrid terms. It reads for the OFM:

$$
\begin{aligned}
& \tau_{c}=\frac{1}{2} \bar{f}\left[\nabla \cdot\left(-2\left(C_{s} \bar{\Delta}\right)^{2}\|\overline{\mathbf{S}}\| \overline{\mathbf{S}}\right)+C_{b}(\overline{\rho \overline{\mathbf{u}} \cdot \nabla \overline{\mathbf{u}}}-\overline{\bar{\rho}} \overline{\overline{\mathbf{u}}} \cdot \overline{\overline{\nabla \mathbf{u}}})\right] \\
& \tau_{i a}=\frac{1}{2} \bar{f}\left[\nabla \cdot\left(-2\left(C_{s} \bar{\Delta}\right)^{2}\|\overline{\mathbf{S}}\| \overline{\nabla C}\right)+C_{b}(\overline{\overline{\mathbf{u}} \cdot \overline{\nabla C}}-\overline{\overline{\mathbf{u}}} \cdot \overline{\overline{\nabla C}})\right]
\end{aligned}
$$


And for the MFA:

$$
\begin{array}{r}
\tau_{c}=\frac{1}{2}\left[\nabla \cdot\left(-2\left(C_{s} \bar{\Delta}\right)^{2}\left\|\overline{\mathbf{S}}_{k}\right\| \bar{\alpha}_{k} \overline{\mathbf{S}}_{k}\right)+C_{b} \rho_{k}\left(\overline{\nabla \cdot\left(\bar{\alpha}_{k} \overline{\mathbf{u}}_{k} \otimes \overline{\mathbf{u}}_{k}\right)}-\nabla \cdot\left(\overline{\bar{\alpha}}_{k} \overline{\overline{\mathbf{u}}}_{k} \otimes \overline{\overline{\mathbf{u}}}_{k}\right)\right)\right](39) \\
\tau_{i a}=\frac{1}{2}\left[\nabla \cdot\left(-2\left(C_{s} \bar{\Delta}\right)^{2}\left\|\overline{\mathbf{S}}_{k}\right\| \overline{\nabla \alpha_{k}}\right)+C_{b} \rho_{k}\left(\overline{\nabla \cdot\left(\bar{\alpha}_{k} \overline{\mathbf{u}}_{k}\right)}-\nabla \cdot\left(\overline{\bar{\alpha}}_{k} \overline{\overline{\mathbf{u}}}_{k}\right)\right)\right](40)
\end{array}
$$

\subsection{Evaluation of LES models for two-phase subgrid terms}

\begin{tabular}{cccccc}
\hline \hline Subgrid term & Smagorinsky & Wale & Bardina & Mixed & ADM \\
\hline \hline One-fluid model & & & & & \\
\hline$\tau_{c}$ & 93 & 108 & 40 & 64 & 17 \\
$\tau_{d}$ & - & - & 72 & - & 32 \\
$\tau_{s t}$ & - & - & 54 & - & 24 \\
$\tau_{i a}$ & 105 & 88 & 62 & 78 & 58 \\
\hline Multi-field model & & & & & \\
\hline$\tau_{c}$ & 100 & 100 & 25 & 59 & 9 \\
$\tau_{d}$ & - & - & 43 & - & 20 \\
$\tau_{s t}$ & - & - & 44 & - & 21 \\
$\tau_{i a}$ & 100 & 100 & 28 & 59 & 11 \\
\hline \hline
\end{tabular}

Table 7: Average relative error (in \%) of the modeled two-phase LES subgrid terms compared to the filtered DNS subgrid terms. Both one-fluid and multifield models are considered. Filter ${ }^{-}$is applied to DNS, with a width of the low-pass filtering operator $G$ equal to 2 cells in each direction.

Thanks to DNS simulations, the two-phase LES subgrid terms can be directly calculated by applying filter $G$ to the various variables of the phase separation problem. Moreover, the various LES models applied to $\tau_{c}, \tau_{d}, \tau_{s t}$ and $\tau_{i a}$ can also be calculated with the DNS results. The main objective of the present section is to evaluate the representativeness of LES models presented in the previous section compared to filtered DNS subgrid terms. Results are presented in figure 5 in terms of correlation between a given model (Smagorinsky, Wale, Bardina, Mixed, ADM), for a given subgrid LES term, i.e. $\tau_{c}, \tau_{d}, \tau_{s t}$ and $\tau_{c}$, and the filtered DNS of a corresponding subgrid term. The evaluation of LES models is provided for both OFM and MFA. On a general point of view, it is observed that compared to OFM, the models 

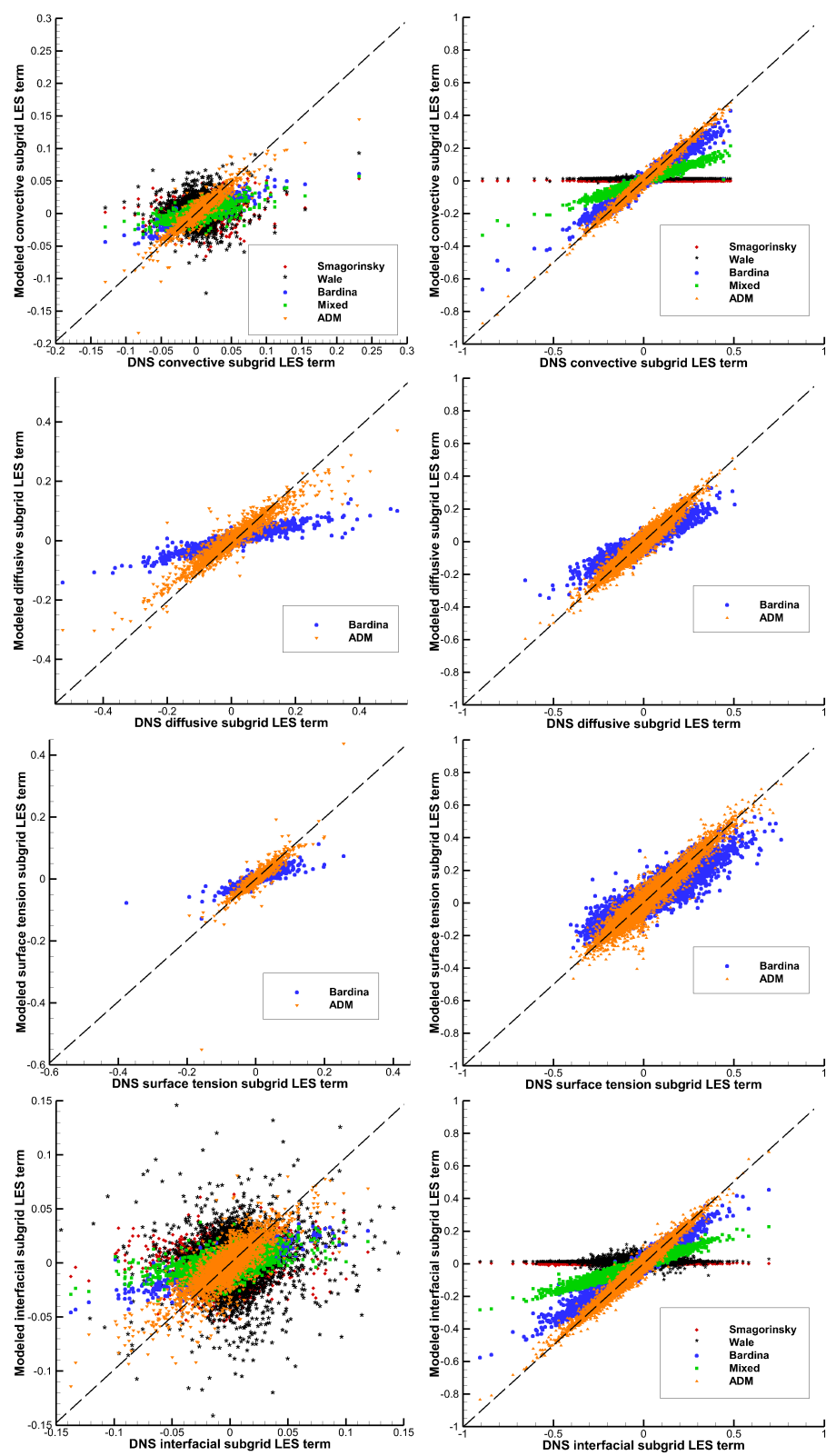

Figure 5: Spatial correlation of two-phase LES subgrid terms - The modeled terms are plotted according to the filtered DNS subgrid terms. A perfect modeling would be on the $\mathrm{y}=\mathrm{x}$ line. Five LES models are considered: Smagorinsky [5], WALE [6], Bardina [7], Mixed model [8] and ADM [9]. Left column is for the one-fluid model whereas right column is for the multi-field approach. 

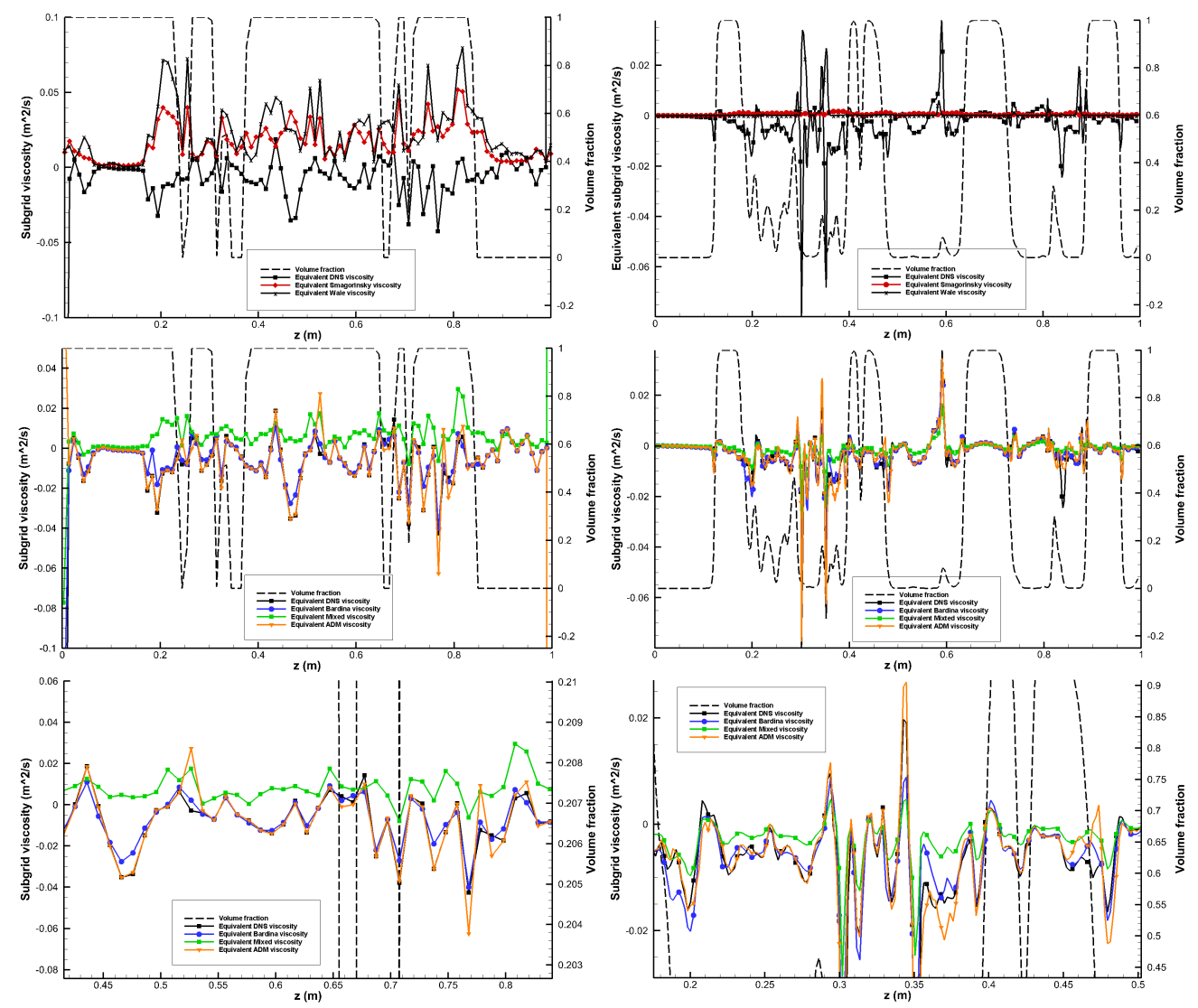

Figure 6: Comparison of equivalent turbulent viscosities obtained with DNS and LES models, i.e. Smagorinsky [5], WALE [6], Bardina [7], Mixed model [8] and ADM [9]. Left column is for OFM and right column for MFA. The bottom line is a zoom on the middle line values. The results are plotted in a vertical line centered on $[x=0.6, y=0.6]$ for OFM and $[x=0.8, y=0.8]$ for MFA. The volume fraction is also plotted to locate interface position as $C=0.5$.

applied to MFA involve less dispersion of the correlation between LES models and filtered DNS. Indeed, the phase average $\alpha_{k}$ is present in all terms for MFA. It tends to eliminate the LES modeling far from the interfacial zone and it damps the gradients or filtering operations in the interfacial zone. On the contrary, in the OFM, all values of the models are a priori sharper and present everywhere, including zones far from the interface. In this case, a larger dispersion of correlation between LES models and filtered DNS subgrid terms is induced. If attention is now paid to the quality of models for a given subgrid term, several observations arise: 
- only structural models are able to model all subgrid terms. Indeed, functional based models such as Smagorinsky, Wale or Mixed do rely on a physical interpretation of the behavior and interaction of under-resolved scales with large scales of turbulence (energy cascade of Kolmogorov, dissipative effect of small scale turbulent structures, ...). They are mainly based on interpretation of kinetic energy and inertial effects in turbulent flows. However, they do not consider the multi-scale character of interfaces when two-phase flows are dealt with. They so can only be applied to advection or inertial terms in conservation equations. This is the reason why functional LES approaches are not possible to be investigated for $\tau_{d}$ and $\tau_{s t}$. If we pretend to perform real LES simulations accounting for all two-phase subgrid terms, using functional models seems to be inappropriate as they do not apply for all subgrid terms. This discussion is valid for both OFM and MFA.

- for the inertial and interfacial terms, all LES models can be compared. It is observed that for both one-fluid and multi-field approaches, the Smagorinsky and Wale models are not representative of filtered DNS terms. The Mixed model is better as it incorporates a contribution from the Bardina model that is working pretty well. The ADM approach is clearly the best for all terms and all modeling approaches (OFM and MFA). The functional based models involve a lot of dispersion and they do not respect the $y=x$ slope that should be obtained if a perfect correlation between LES models and filtered DNS will be reached. The interesting point here is that ADM based models (and also Bardina but with poorer results) are working for all subgrid terms and all two-phase flow modeling approaches (OFM and MFA). The average percentage error that is obtained for all models, all subgrid terms and all two-phase flow models is given in table 7 . It can be clearly inferred that Smagorinsky and Wale model are not suitable for two-phase subgrid terms, even concerning inertia. The representativeness of Mixed model is better than Smagorinsky, as it incorporates a part of Bardina modeling. The Bardina model is performing quite nicely for all subgrid terms. It behaves less well for $\tau_{d}$ and $\tau_{i a}$ when OFM is considered whereas its quality is worse for $\tau_{d}$ and $\tau_{s t}$ with regard to MFA. It could be imagined to play on the Bardina constant $C_{b}$ so as to improve the performance of this model and to get closer to $y=x$ curve on the correlation plots. Finally, the ADM model is the best for all subgrid terms and all two-phase flow modeling approaches. The maximum error with MFA is at least $20 \%$ whereas this error is in the range 17 to $32 \%$ for OFM, with a peak of error of $58 \%$ for $\tau_{i a}$.

If true LES were simulated, the use of LES models for convective subgrid 
terms will be equivalent to add a turbulent viscosity in the viscous term of the momentum equations. It is interesting to evaluate the equivalent turbulent viscosity resulting from the use of a given LES model, compared to the equivalent viscosity that the filtering of the DNS provides when the two-phase convective LES subgrid term is considered. Classically [16,31], we can write the turbulent viscosity $\mu_{t}$ for the OFM as follows:

$$
\mu_{t}=\frac{\tau_{c}: \overline{\nabla \mathbf{u}}}{\overline{\mathbf{S}}: \overline{\nabla \mathbf{u}}}
$$

And for the MFA:

$$
\mu_{t}=\alpha_{1} \frac{\tau_{c, 1}: \nabla \mathbf{u}_{1}}{\overline{\mathbf{S}_{1}: \overline{\nabla \mathbf{u}_{1}}}}+\alpha_{2} \frac{\tau_{c, 2}: \nabla \mathbf{u}_{2}}{\overline{\mathbf{S}_{2}: \overline{\mathbf{u}_{2}}}}
$$

Equivalent LES turbulent viscosities compared to equivalent filtered DNS one are plotted in figure 6. For OFM, the instantaneous turbulent viscosities are extracted in a vertical slice starting from point $(0.6,0.6,0)$ whereas for MFA, the strating of the slice is $(0.8,0.8,0)$. A time $t^{*} \approx 3$ is considered for the peak of enstrophy. The instantaneous simulations of OFM and MFA at a given time are not exhibiting the same interfacial and flow structures. The slices for turbulent viscosities have been chosen in order to cut different times the interface. If we focus on the equivalent DNS viscosity that we should obtained with LES models, we observe that negative values are obtained. This have previously been been reported by $[31,32]$. This important feature of the effect of inertial subgrid term cannot be represented by functional LES approaches as they are intrinsically built on positive terms. This is illustrated in the line of figure 6. This way, Smagorinsky and Wale models are again discriminated against structural ones. If we now compare Bardina, ADM and Mixed models, we notice that the first two are much better as expected. Concerning equivalent turbulent viscosities, we observe that ADM is globally nearer to DNS than Bardina, even if locally, Bardina can exhibit closer values to DNS reference.

To conclude on LES modeling of two-phase subgrid LES terms, it has been demonstrated that the structural approaches are the correct way of building models. The ADM formalism allows to recover the negative values of equivalent turbulent viscosities in the good zones of the flow and they respect the behavior of filtered DNS subgrid terms with less than $30 \%$ discrepancy $\left(50 \%\right.$ for $\left.\tau_{i a}\right)$. We have to keep in mind that ADM modeling allows to reconstruct the fields between the explicit filter that we have and the implicit filter brought by the simulation mesh. In real LES, an additive relaxation 
term will have to be incorporated in the momentum equations [9] in order to obtain the effect of under-resolved scales smaller than the implicit filter size.

\section{Summary and future work}

Based on direct numerical simulation of two-phase flow with resolved scale interfaces, i.e. a phase inversion problem, a priori filtering of two-phase subgrid LES terms has been investigated. The one-fluid model and multi-field approaches were considered and filtered. In addition, different LES models taken from the turbulent single-phase flow literature were utilized to propose functional and structural LES models for two-phase LES subgrid terms. The order of magnitude of all a priori filtered subgrid terms and LES models for these terms were compared to filtered DNS values for the first time on a same two-phase problem. The formulation of LES models for all subgrid terms was also proposed and discussed for the first time, in particular for a multi-field approach.

Concerning a priori filtering, the main conclusion is that the inertia term is not predominant in two-phase flows with fragmentation and rupture of interface that exhibit a kind of atomization process. This conclusion is different from that of the studies of [3,29-32]. As soon as small-scale interfacial structures are generated, correlation terms induced by the curvature in the subgrid surface tension terms or between the local interface presence and the velocity are predominant against inertial terms. The diffusive viscous term is always small in our phase inversion problem, whatever the two-phase flow model or the kind of filtering.

Five different LES models applied to two-phase subgrid LES terms have been compared against filtered DNS results. Two classes of models have been considered, the functional (Smagorinsky, Wale) and the structural (Bardina, ADM) approaches. A combined functional-structural model, called Mixed Bardina-Smagorinsky, have also been investigated. Conclusion are clear for both OFM and MFA: functional LES modeling do not correlate to filtered DNS results whereas structural approaches do. Bardina and ADM are clearly the good LES framework to consider for two-phase flows with resolved scale interfaces. ADM is clearly better than Bardina in our study. It has to be noticed that we do not have trigered the Bardina constant to try to improve the Bardina results. This could be usefull to try to get closer to ADM. The improvement of Bardina, if possible, would be a nice point as ADM of $7^{\text {th }}$ is clearly more CPU time consuming. The Mixed approach, as expected, is bet- 
ter than functional models and worse than structural LES techniques. For the first time, we have been able to demonstrate that a common LES framework could be used for modeling all two-phase subgrid terms with resolved scale interfaces, i.e. the approximate deconvolution method. The main interest of structural approaches is their capability of representing negative equivalent turbulent viscosities whereas functional models are generating intrinsically positive viscosities. We have shown that filtered equivalent DNS viscosities are clearly negative in specific zones of the flow, not only near the interface. This negative feature is expected from LES models.

Future works will be oriented in two ways:

- performing a priori LES filtering and model comparisons on finer DNS meshes. A $2048^{3}$ grid is under consideration for phase inversion. The same analysis will be used on other two-phase flows with separated phases, such as the atomization of a liquid sheet in a HIT flow [44]. The idea is to control the turbulence forcing and to vary the properties of the fluid and interface properties to try to extract general conclusions on LES modeling frameworks for two-phase flows.

- investigating real ADM LES with both OFM and MFA. A coarse LES grid will be used in this case. The idea is to see if compared to DNS, the LES are able to capture the main features of the flow. The other important point is to compare implicit LES, i.e. LES without explicit modeling of the subgrid terms, and ADM models for all two-phase subgrid terms. In particular, the role of the introduction of a relaxation term [9] in the momentum equations will be discussed and evaluated compared to subgrid LES contributions. The starting test problem could be the phase inversion benchmark, for which different $R e$ and $W e$ can be chosen in order to adjust inertial and capillary effects compared to viscous and gravity contributions. The interest of this case is that DNS can be generated and used for LES comparisons.

\section{Acknowledgements}

The authors are grateful for access to the computational facilities of the French CINES (National computing center for higher education) and CCRT (National computing center of CEA) under project number x20152b6115 and x20162b6115. The Agence Nationale de la Recherche (ANR) through the MODEMI project is associated to this work. Thank EDF for the financial support it has given to this work. 


\section{References}

[1] S. Vincent, J.-P. Caltagirone, and D. Jamet. Test case $\mathrm{n}^{\circ} 15$ : phase inversion in a closed box (pn, pe). Multiphase Sci. Technol., 6:101-104, 2004 .

[2] J. Larocque, S. Vincent, P. Lubin, D. Lacanette, and J.-P. Caltagirone. Parametric study of LES subgrid terms in a turbulent phase separation flow. Int. J. Heat Fluid Flow, 31:536-544, 2010.

[3] S. Vincent, D. Lacanette, J. Larocque, A. Toutant, P. Lubin, and P. Sagaut. Direct numerical simulation of phase separation and a priori two-phase LES filtering. Comput. Fluids, 37:898-906, 2008.

[4] S. Vincent, L. Osmar, J.-L. Estivalezes, S. Zaleski, F. Auguste, W. Aniszewski, Y. Ling, T. Ménard, A. Pedrono, J. Magnaudet, J.P. Caltagirone, and A. Berlemont. A phase inversion benchmark for multiscale multiphase flows. submitted to J. Comput. Phys., 2016.

[5] J. Smagorinsky. General circulation experiments with the primitive equations. i : The basics experiment. Month. Weather Rev., 91:99-165, 1963.

[6] F. Nicoud and F. Ducros. Subgrid-scale stress modelling based on the square of the velocity gradient tensor. Flow Turb. Comb., 62:183-200, 1999.

[7] J. Bardina, J.H. Ferziger, and W.C. Reynolds. Improved subgrid scale models for large eddy simulation. AIAA Paper, 80:1357, 1980.

[8] J. Bardina, J.H. Ferziger, and W.C. Reynolds. Improved turbulence models based on large eddy simulation of homogeneous, incompressible, turbulent flows. Report TF-19, Thermosciences Division, Det. Mechanical engineering, Standford University, 1983.

[9] N.A. Adams and S. Stolz. A subgrid-scale deconvolution approach for shock capturing. J. Comput. Phys., 178:391-426, 2002.

[10] C.T. Crowe. Multiphase flow handbook. CRC Press, Taylor and Francis, 2005 .

[11] S. Vincent, J.-P. Caltagirone, and D. Jamet. Test-case $\mathrm{n}^{\circ} 15$ : phase inversion in a closed box (pc). Multiphase Sci. Technol., 16:101-104, 2004 . 
[12] C. Kleinstreuer. Two-phase flow: theory and applications. Taylor and Francis, 2003.

[13] R. Lebas, T. Ménard, P.A. Beau, A. Berlemont, and F.X. Demoulin. Numerical simulation of primary break-up and atomization: DNS and modelling study. Int. J. Multiphase Flow, 35:247-260, 2009.

[14] D. Zuzio, J.-L Estivalezes, P. Villedieu, and G. Blanchard. Numerical simulation of primary and secondary atomization. C. R. Mécanique, 341:15-25, 2013.

[15] P. Trontin, S. Vincent, J.-L. Estivalezes, and J.-P. Caltagirone. Direct numerical simulation of a freely decaying turbulent interfacial flow. Int. J. Multiphase Flow, 36:891-907, 2010.

[16] P. Sagaut. Large Eddy Simulation for incompressible flows - An introduction. Springer Verlag, 1998.

[17] I. Kataoka. Local instant formulation of two-phase flow. Int. J. Multiphase Flow, 12:745-758, 1986.

[18] R. Scardovelli and S. Zaleski. Direct numerical simulation of free-surface and interfacial flow. Annu. Rev. Fluid Mech., 31:567-603, 1999.

[19] A. Prosperetti and G. Tryggvason. Computational methods for multiphase flows. Cambridge University Press, 2007.

[20] C.W. Hirt and B.D. Nichols. Volume of Fluid (VOF) Method for the Dynamics of Free Boundaries. J. Comput. Phys, 39:201-225, 1981.

[21] S. Osher and R. Fedkiw. Level set methods: An overview and some recent results. J. Comput. Phys., 169:463-502, 2001.

[22] G. Tryggvason, R. Scardovelli, and S. Zaleski. Direct numerical simulations of gas-liquid multiphase flows. Cambridge University Press, 2011.

[23] R. Denèfle, S. Mimouni, J.-P. Caltagirone, and S. Vincent. Multifield hybrid approach for two-phase flow modeling - part 1: Adiabatic flows. Comput. Fluids, 113:106-111, 2015.

[24] S. Fleau, S. Mimouni, N. Mérigoux, and S. Vincent. Simulations of twophase flows with a multifield approach. In Proceedings of Computational Heat Transfer conference CHT-15, May 2015, New Brunswick, USA, 2015 . 
[25] S. Fleau, S. Mimouni, N. Mérigoux, and S. Vincent. Validation of a multifield approach for the simulations of two-phase flows. Comput. Thermal Sci., 7:1-17, 2016.

[26] D. Jacqmin. Calculation of Two-Phase Navier-Stokes Flows Using Phase-Field Modeling. J. Comput. Phys, 155:96-127, 1999.

[27] P. Sagaut and C. Cambon. Homogeneous turbulence dynamics. Cambridge University Press, 2008.

[28] P. Liovic and D. Lakehal. Multi-physics treatment in the vicinity of arbitrarily deformable gas-liquid interfaces. J. Comput. Phys., 222:504$535,2007$.

[29] P. Liovic and D. Lakehal. Interface-turbulence interactions in large-scale bubbling processes. Int. J. Heat Fluid Flow, 28:127-144, 2006.

[30] J. Chesnel, Reveillon J., F.X. Demoulin, and T. Menard. Subgrid modeling of liquid atomization. In 6th International Conference on Multiphase Flow, Leipzig, Germany, 2007.

[31] E. Labourasse, D. Lacanette, A. Toutant, P. Lubin, S. Vincent, O. Lebaigue, J.-P. Caltagirone, and P. Sagaut. Towards large eddy simulation of isothermal two-phase flows: Governing equations and $a$ priori tests. Int. J. Multiphase Flow, 33:1-39, 2007.

[32] A. Toutant, E. Labourasse, O. Lebaigue, and O. Simonin. Dns of the interaction between a deformable buoyant bubble and a spatially decaying turbulence: a priori tests for les two-phase flow modelling. Comput. Fluids, 37:877-886, 2008.

[33] W. Aniszewski, A. Boguslawski, M. Marek, and A. Tyliszczak. A new approach to sub-grid surface tension for les of two-phase flows. J. Comput. Phys., 231:7368-7379, 2012.

[34] W. Aniszewski, T. Ménard, and M. Marek. Volume of fluid (VOF) type advection methods in two-phase flow: A comparative study. Comput. Fluids, 97:52-73, 2014.

[35] G. Tomar, D. Fuster, S. Zaleski, and S. Popinet. Multiscale simulations of primary atomization. Comput. Fluids, 39:1864-1874, 2010.

[36] J.M. Delhaye. Jump conditions and entropy sources in two-phase systems. Local instant formulation. Int. J. Multiphase Flow, 1:395-409, 1974 . 
[37] J.U. Brackbill, D.B. Kothe, and C. Zemach. A continuum method for modeling surface tension. J. Comput. Phys., 100:335-354, 1992.

[38] A.J. Chorin. Numerical solution of the Navier-Stokes equations. Math. Comput., 22:745-762, 1968.

[39] R. Temam. Sur l'approximation de la solution des équations de NavierStokes par la méthode des pas fractionnaires. Arch. Ration. Mech. Anal., 33:377-385, 1969.

[40] R. Fedkiw, T. Aslam, B. Merriman, and S. Osher. A non-oscillatory Eulerian approach to interfaces in multimaterials flows (the ghost fluid method). J. Comput. Phys., 152:457-492, 1999.

[41] Chi-Wang Shu. Essentially Non-Oscillatory and Weighted Essentially Non-Oscillatory Schemes for Hyperbolic Conservation Laws. NASA (as NASA/CR-97-206253 report), 1997.

[42] C.W. Shu and S. Osher. Efficient implementation of essentially nonoscillatory shock-capturing schemes. J. Comput. Phys., 77:439-471, 1988.

[43] M. Sussman, E. Fatemi, P. Smereka, and S. Osher. Improved level set method for incompressible two-phase flows. Comput. Fluids, 27:663-680, 1998.

[44] P. Trontin, S. Vincent, J.-L. Estivalezes, and J.-P. Caltagirone. Detailed comparisons of front-capturing methods for turbulent two-phase flow simulations. Int. J. Numer. Meth. Fluids, 56:1543-1549, 2008.

[45] D. Zuzio and J.-L. Estivalezes. An efficient block parallel AMR method for two phase flow simulations. Comput. Fluids, 44:339-357, 2011.

[46] M. Ishii. Thermo-fluid dynamics, theory of two-phase flow,. Eyrolles, 1975.

[47] S. Mimouni, R. Denèfle, S. Fleau, and S. Vincent. Multifield approach and interface locating method for two-phase flows in nuclear power plant. In SimHydro 2014: Modelling of rapid transitory flows, 11-13 June, Sophia-Antipolis, 2014.

[48] Y. Bartosiewicz. A first assessment of the NEPTUNE_CFD code: Instabilities in a stratified flow comparison between the vof method and a two-field approach. Int. J. Heat and Fluid Flow, 29:460-478, 2008. 
[49] S. Patankar and D. Spalding. A calculation procedure for heat, mass and momentum transfer in three-dimensional parabolic flows. Int. J. Heat Mass Transf., 15:1787-1806, 1975.

[50] S. Fleau, S. Mimouni, and S. Vincent. Conservative implementation of the interface sharpening equation within a multifield approach. under correction in Comput. Fluids, 2015.

[51] E. Olsson and G. Kreiss. A conservative level set method for two phase flow. J. Comput. Phys., 210:225-246, 2005.

[52] L. Strubelj. Numerical simulations of stratified two-phase flows with twofluid model and interface sharpening. PhD thesis, University of Ljublana, 2009 .

[53] O. Vasilyev, T.S. Lund, P. Moin, and K. Aksellvoll. A general class of commmutative filters for les in complex geometries. J. Comput. Phys., 146:82-104, 1998.

[54] Y.M. Dakhoul and K.W. Bedford. Improved averaging method for turbulent flow simulation. part 1: theoretical development and application to burger's transort equation. Int. J. Numer. Meth. Fluids, 6:49-64, 1986.

[55] Y.M. Dakhoul and K.W. Bedford. Improved averaging method for turbulent flow simulation. part 2: calculation and verification. Int. J. $\mathrm{Nu}$ mer. Meth. Fluids, 6:65-82, 1986.

[56] M. Tavares, S. Vincent, M. Ould-Rouiss, and J.-L. Estivalezes. A priori study for the modeling of LES subgrid scale terms. In Proceedings of the $4^{\text {th }}$ Turbulence and Interactions conference TI2015, November 2015, Cargèse, France, Springer, 2016.

[57] S. Fleau, S. Vincent, and S. Mimouni. LES modeling with a multifield approach. In Proceedings of the $4^{\text {th }}$ Turbulence and Interactions conference TI2015, November 2015, Cargèse, France, Springer, 2016.

[58] P. Sagaut and M. Germano. On the filtering paradigm for les of flows with discontinuities. J. Turbulence, 6:1-9, 2005.

[59] D. Lacanette, A. Gosset, S. Vincent, J.-M. Buchlin, and E. Arquis. Macroscopic analysis of gas-jet wiping: Numerical simulation and experimental approach. Phys. Fluid, 18:042103:1-15, 2006. 
[60] J. Larocque, N. Rivière, S. Vincent, D. Reungoat, J.-P. Fauré, J.-P. Héliot, J.-P. Caltagirone, and M. Moreau. Macroscopic analysis of a turbulent round liquid jet impinging on an air/water interface in a confined medium. Phys. Fluid, 21:065110:1-21, 2009.

[61] I. Calmet and J. Magnaudet. High-schmidt number mass transfer through turbulent gas-liquid interfaces. Int. J. Heat Fluid Flow, 19:522532, 1998.

[62] I. Calmet and J. Magnaudet. Statistical structure of high-reynoldsnumber turbulence close to the free surface of an open-channel flow. $J$. Fluid Mech., 474:355-378, 2003.

[63] I. Calmet and J. Magnaudet. Large-eddy simulation of high-Schmidtnumber mass transfer in a turbulent channel flow. Phys. Fluids, 9:438$455,1997$. 